\title{
FOURIER EXPANSIONS OF GL(2) NEWFORMS AT VARIOUS CUSPS
}

\author{
DORIAN GOLDFELD, JOSEPH HUNDLEY, AND MIN LEE
}

\begin{abstract}
This paper studies the Fourier expansion of Hecke-Maass eigenforms for $G L(2, \mathbb{Q})$ of arbitrary weight, level, and character at various cusps. Translating well known results in the theory of adelic automorphic representations into classical language, a multiplicative expression for the Fourier coefficients at any cusp is derived. In general, this expression involves Fourier coefficients at several different cusps. A sufficient condition for the existence of multiplicative relations among Fourier coefficients at a single cusp is given. It is shown that if the level is 4 times (or in some cases 8 times) an odd squarefree number then there are multiplicative relations at every cusp. We also show that a local representation of $G L\left(2, \mathbb{Q}_{p}\right)$ which is isomorphic to a local factor of a global cuspidal automorphic representation generated by the adelic lift of a newform of arbitrary weight, level $N$, and character $\chi(\bmod N)$ cannot be supercuspidal if $\chi$ is primitive. Furthermore, it is supercuspidal if and only if at every cusp (of width $m$ and cusp parameter $=0$ ) the $m p^{\ell}$ Fourier coefficient, at that cusp, vanishes for all sufficiently large positive integers $\ell$. In the last part of this paper a three term identity involving the Fourier expansion at three different cusps is derived.
\end{abstract}

\section{Contents}

1. Introduction

2. Newforms and Hecke operators for $\Gamma_{0}(N)$

3. Whittaker functions for the adelic lift of a newform

4. A classical consequence of the tensor product theorem

5. Remarks on choices of $\mathfrak{a}$ and $\gamma_{\mathfrak{a}}$

6. On Stark's Question

7. A classical interpretation of the Jacquet-Langlands criterion for supercuspidality

8. Toward a classical proof of Theorem 15

References

\section{INTRODUCTION}

This paper was inspired by the following question raised by Harold Stark.

When do the Fourier coefficients at a cusp of a classical newform for $G L(2)$ satisfy multiplicative relations?

By a celebrated theorem of Hecke the Fourier coefficients at the cusp $\infty$ satisfy multiplicative relations. Similar results regarding the Fourier coefficients at an arbitrary cusp, for a newform on $\Gamma_{0}(N)$, have been proven by Asai when the level $N$ is squarefree [1]. The proof is, roughly, that if $N$ is squarefree, then the cusps are represented by the quotients $1 / t$ with $t$ running over the positive divisors of $N$, and the corresponding Fricke involution $W_{t}$ maps the cusp $\infty$ to $1 / t$ and, at the same time, acts as an involution on the space of newforms commuting with all Hecke operators. Kojima was able to obtain a similar result by another method in the case when the level is $4 \mathrm{q}$ where $\mathrm{q}$ is a prime [18. Further, Asai's reasoning may be applied to general $N$, yielding multiplicative relations at any cusp which is related to $\infty$ by a Fricke involution. (Such cusps are in one-to-one correspondence with divisors $d$ of $N$ such that $\operatorname{gcd}(d, N / d)=1$.) In the general case, very little was known about the Fourier coefficients at cusps which can not be mapped to $\infty$ by a Fricke involution, at least in classical terms.

The first author was partially supported by NSF grant 1001036. The second author was supported by NSA grant MSPF08Y-172. 
Many of the results of this paper are probably well known to experts in the theory of adelic automorphic representations, but the translation back to classical language is not so easy. We have been unable to find anything on this topic in the literature (beyond the aforementioned results of Asai and Kojima) so we thought it would be useful to write up our explicit results.

The celebrated tensor product theorem [7] (see also [4 §3.4) states that an irreducible adelic automorphic representation factors as a tensor product of local representations. Our theorem 15 may be viewed as a translation of the tensor product theorem into a statement about Fourier coefficients of Maass forms. In theorem 19, we discuss the special case of prime power level in greater detail. These theorems give a partial answer to Stark's question, by providing a multiplicative expression for the Fourier coefficients at an arbitrary cusp. However, in most cases this expression will involve Fourier coefficients at several other cusps. Theorem 35 gives a sufficient condition for multiplicative relations involving Fourier coefficients at a single cusp. As a consequence, we deduce that there are multiplicative relations at every cusp if the level $N$ is equal to 4 times a squarefree odd number. Further, if $N$ is 8 times a squarefree odd number, then theorem 35 will imply multiplicative relations at all cusps except for those of the form $a / b$ with 2||$b$. In proposition 42 we consider such cusps in detail.

When the level of a Maass form is divisible by the square of a prime, one expects more complicated behavior, both classically and representation-theoretically. From the representation-theoretic point of view, this added complexity is in part due to the existence of supercuspidal representations. In Theorem 44, we unpack the classical content of a criterion for a representation of $G L\left(2, \mathbb{Q}_{p}\right)$ to be supercuspidal. This states that an irreducible smooth representation of $G L\left(2, \mathbb{Q}_{p}\right)$ is supercuspidal if and only if its Kirillov model is the Schwartz space of $\mathbb{Q}_{p}^{\times}$([15], Proposition 2.16, [10, Theorem 3). As we show in Theorem 44, this implies that a representation of $G L\left(2, \mathbb{Q}_{p}\right)$ which is isomorphic to the local representation factor of an irreducible automorphic representation of $G L(2, \mathbb{A})$ (generated by the adelic lift of a classical Hecke-Maass newform) is supercuspidal if and only if the $m_{\mathfrak{a}} p^{\ell}$ Fourier coefficient vanishes for all sufficiently large integers $\ell$ for each cusp $\mathfrak{a} \in \mathbb{Q} \cup\{\infty\}$. Here $m_{\mathfrak{a}}$, also called the width of the cusp, is an integer given by (4). It is well-known (see [3], [8] and also Proposition 5.1 of [23]) that every supercuspidal representation of $G L\left(2, \mathbb{Q}_{p}\right)$ can be realized as a component of a global irreducible cuspidal automorphic representation of $G L(2, \mathbb{A})$, and that every such representation may be associated to a normalized Hecke-Maass newform. Theorem 44 gives a criterion for recognizing those Hecke-Maass newforms which are connected with supercuspidals in this way. By combining this with a result from the classical side, we deduce, in Corollary 45 that a local representation of $G L\left(2, \mathbb{Q}_{p}\right)$ cannot be supercuspidal if it isomorphic to a local factor of a global cuspidal automorphic representation generated by the adelic lift of a Hecke-Maass newform of weight $k$, level $N$ and character $\chi(\bmod N)$ if $\chi$ is a primitive character. This shows that supercuspidal representations can only arise from Hecke-Maass newforms of level $N$ with imprimitive characters $(\bmod N)$. In this connection, we would also like to mention a result of Casselman (see [5]), which implies that supercuspidal representations of $G L\left(2, \mathbb{Q}_{p}\right)$ can only arise from Masss-Hecke newforms of level $N$ such that $p^{2} \mid N$. For more detail see the remark which follows the proof of Corollary 45 .

Theorem 15 is a classical statement. It is natural to ask whether it is possible to prove it classically. This problem is considered in section 8. Although we do not recover the full strength of theorem 15, we do obtain relations between the Fourier coefficients at different cusps for Maass forms of arbitrary weight level and character, using a purely classical approach adapted from that employed by Kojima in the case $N=4 q$. The main result is given in Theorem 49 where a three term relation (involving three different cusps) is obtained.

Acknowledgements: The authors would like to thank Hervé Jacquet, Muthu Krishnamurthy, Omer Offen, Scott Ahlgren, and Jeremy Rouse for some helpful conversations.

\section{Newforms ANd Hecke operators For $\Gamma_{0}(N)$}

In this section we briefly review the theory of newforms and Hecke operators for the group $\Gamma_{0}(N)$. We fix an integer $k$ (called the weight), an integer $N \geq 1$ (called the level), and a Dirichlet character $\chi:(\mathbb{Z} / N \mathbb{Z})^{\times} \rightarrow \mathbb{C}^{\times}$. Let $\mathfrak{h}$ denote the upper half plane. For any function $f: \mathfrak{h} \rightarrow \mathbb{C}$, and any matrix 
$\gamma \in G L(2, \mathbb{R})$ of positive determinant, define the slash operator

$$
\left(\left.f\right|_{k} \gamma\right)(z):=\left(\frac{c z+d}{|c z+d|}\right)^{-k} f\left(\frac{a z+b}{c z+d}\right)
$$

and the character $\tilde{\chi}: \Gamma_{0}(N) \rightarrow \mathbb{C}^{\times}$defined by

$$
\tilde{\chi}\left(\left(\begin{array}{ll}
a & b \\
c & d
\end{array}\right)\right):=\chi(d) .
$$

An automorphic function of weight $k$, and character $\chi$ for $\Gamma_{0}(N)$ is a smooth function $f: \mathfrak{h} \rightarrow \mathbb{C}$ which satisfies the automorphy relation

$$
\left(\left.f\right|_{k} \gamma\right)(z)=\tilde{\chi}(\gamma) f(z), \quad(z \in \mathfrak{h})
$$

for all $\gamma \in \Gamma_{0}(N)$.

Now fix $\nu \in \mathbb{C}$. A Hecke-Maass form of weight $k$, type $\nu$, level $N$ and character $\chi(\bmod N)$ is an automorphic cuspidal function of weight $k$, level $N$ and character $\chi$ which has moderate growth and which is also an eigenfunction of the weight $k$ Laplace operator,

$$
\Delta_{k}=-y^{2}\left(\frac{\partial^{2}}{\partial x^{2}}+\frac{\partial^{2}}{\partial y^{2}}\right)+i k y \frac{\partial}{\partial x},
$$

with eigenvalue $\nu(1-\nu)$.

Let $f$ be an automorphic function of weight $k$, level $N$ and character $\chi$. For any positive integer $n$, the Hecke operator (twisted by a character $\chi$ ) is denoted $T_{n}^{\chi}$, and is defined by

$$
\left(T_{n}^{\chi} f\right)(z):=\frac{1}{\sqrt{n}} \sum_{a d=n, a, d>0} \chi(a) \sum_{b=0}^{d-1}\left(\left.f\right|_{k}\left(\begin{array}{ll}
a & b \\
0 & d
\end{array}\right)\right)(z) .
$$

Note that every Dirichlet character modulo $N$, including the ("trivial") principal character, vanishes on integers which are not relatively prime to $N$. This will kill some of the terms in the definition of $T_{n}^{\chi}$ when $(n, N) \neq 1$. Notably, if $q$ is a prime dividing $N$, then

$$
\left(T_{q}^{\chi} f\right)(z):=\frac{1}{\sqrt{q}} \sum_{b=0}^{q-1}\left(\left.f\right|_{k}\left(\begin{array}{ll}
1 & b \\
0 & q
\end{array}\right)\right)(z),
$$

which coincides with the operator denoted $U_{q}^{*}$ in 2 .

Cusps are defined to be elements of $\mathbb{Q} \cup\{\infty\}$. The group $S L(2, \mathbb{Z})$ permutes the cusps transitively. Thus, given a cusp $\mathfrak{a}$ it is possible to choose a matrix $\gamma_{\mathfrak{a}} \in S L(2, \mathbb{Z})$ such that $\gamma_{\mathfrak{a}} \infty=\mathfrak{a}$. The matrix $\gamma_{\mathfrak{a}}$ is unique up to an element of the stabilizer, $\Gamma_{\infty}$, of $\infty$ on the right. This group is given explicitly by

$$
\Gamma_{\infty}=\left\{\left(\begin{array}{cc}
\epsilon & n \\
0 & \epsilon
\end{array}\right) \mid \epsilon \in\{ \pm 1\}, n \in \mathbb{Z}\right\},
$$

and is contained in the group $\Gamma_{0}(N)$ for every $N$. In particular, $\gamma_{\mathfrak{a}} \delta \gamma_{\mathfrak{a}}^{-1}$ is independent of the choice of $\gamma_{\mathfrak{a}}$ for each $\delta \in \Gamma_{\infty}$. Let $\Gamma_{\mathfrak{a}}=\left\{\gamma \in \Gamma_{0}(N) \mid \gamma \mathfrak{a}=\mathfrak{a}\right\}$. Then $\gamma_{\mathfrak{a}}^{-1} \Gamma_{\mathfrak{a}} \gamma_{\mathfrak{a}}$ is a subgroup of finite index in $\Gamma_{\infty}$, and contains the scalar matrix -1 . As such it is the product of the group of order 2 generated by -1 and an infinite cyclic group generated by $\left(\begin{array}{cc}1 & m_{\mathfrak{a}} \\ 0 & 1\end{array}\right)$ for some positive integer $m_{\mathfrak{a}}$. This integer $m_{\mathfrak{a}}$ may be characterized as the least positive power of $\gamma_{\mathfrak{a}}\left(\begin{array}{lll}1 & 1 \\ 0 & 1\end{array}\right) \gamma_{\mathfrak{a}}^{-1}$ which is in $\Gamma_{0}(N)$, and as such is independent of the choice of $\gamma_{\mathfrak{a}}$. Let

$$
\sigma_{\mathfrak{a}}=\gamma_{\mathfrak{a}}\left(\begin{array}{cc}
\sqrt{m_{\mathfrak{a}}} & 0 \\
0 & \sqrt{m_{\mathfrak{a}}}-1
\end{array}\right)
$$

then $\sigma_{\mathfrak{a}}$ has the following key properties:

$$
\sigma_{\mathfrak{a}} \infty=\mathfrak{a}, \quad \sigma_{\mathfrak{a}}^{-1} \Gamma_{\mathfrak{a}} \sigma_{\mathfrak{a}}=\Gamma_{\infty} .
$$

The matrix $\sigma_{\mathfrak{a}}\left(\begin{array}{ll}1 & 1 \\ 0 & 1\end{array}\right) \sigma_{\mathfrak{a}}^{-1} \in \Gamma_{\mathfrak{a}}$ is independent of the choice of $\sigma_{\mathfrak{a}}$. We denote this element by $g_{\mathfrak{a}}$. It generates $\Gamma_{\mathfrak{a}}$ together with the scalar matrix -1 . We define the cusp parameter $0 \leq \mu_{\mathfrak{a}}<1$ determined by the condition

$$
\widetilde{\chi}\left(g_{\mathfrak{a}}\right)=e^{2 \pi i \mu_{\mathfrak{a}}} .
$$


Let $f$ be an automorphic function of weight $k$, level $N$ and character $\chi$. Then $f$ is cuspidal if

$$
\int_{0}^{1}\left(\left.f\right|_{k} \sigma_{\mathfrak{a}}\right)(x+i y) d x=0
$$

for any cusp a. If $f$ is a Maass form of weight $k$, type $\nu$, level $N$, and character $\chi$, and $m$ is a positive integer, then $g(z):=f(m z)$ is a Maass form of weight $k$, type $\nu$, level $m N$ and character $\chi \cdot \chi_{0, m N}$, where $\chi_{0, m N}$ is the principal character modulo $m N$. It may be regarded as a form of weight $k$ and type $\nu$ for $\Gamma_{0}(M)$, where $M$ is any multiple of $m N$. Forms which arise in this manner are said to be old, or to be oldforms. A Maass cusp form $f$ of weight $k$, type $\nu$, level $N$, and character $\chi$ is said to be new or a newform if it is orthogonal (with respect to the Petersson inner product) to every oldform. The main result of Atkin-Lehner theory [2] is that the space of newforms has a basis where each basis element is an eigenfunction of all the Hecke operators. Such newforms are called Maass-Hecke newforms.

We remark that a Maass form of level $N$ is a Masss-Hecke newform if and only if it is an eigenfunction of $T_{p}^{\chi}$ for every prime $p$, and of the operator $f(z) \mapsto f(\overline{-1 / N \bar{z}})$. In the holomorphic case, this follows from Theorem 9 of [20]. The same proof works in the non-holomorphic case.

It was shown in [21], 22] that a newform of weight $k$, level $N$, and character $\chi(\bmod N)$ for $\Gamma_{0}(N)$ has a Fourier-Whittaker expansion at every cusp. The expansion takes the following explicit form.

Proposition 6. (Fourier-Whittaker expansion at a cusp) Let $f$ be a Maass form of weight $k$, type $\nu$, level $N$, and character $\chi(\bmod N)$ for $\Gamma_{0}(N)$. Let $\mathfrak{a} \in \mathbb{Q} \cup\{\infty\}$ be a cusp and let $\sigma_{\mathfrak{a}}, \mu_{\mathfrak{a}}$ be as in (4), (5), respectively. Then

$$
\left(\left.f\right|_{k} \sigma_{\mathfrak{a}}\right)(z)=\sum_{n+\mu_{\mathfrak{a}} \neq 0} A(\mathfrak{a}, n) W_{\frac{\operatorname{sgn}(n) k}{2}, \nu-\frac{1}{2}}\left(4 \pi\left|n+\mu_{\mathfrak{a}}\right| \cdot y\right) e^{2 \pi i\left(n+\mu_{\mathfrak{a}}\right) x}, \quad(z=x+i y \in \mathfrak{h})
$$

where $A(\mathfrak{a}, n) \in \mathbb{C}$ is called the $n^{\text {th }}$ Fourier coefficient at the cusp $\mathfrak{a}$ and

$$
W_{\alpha, \nu}(y)=\frac{y^{\nu+\frac{1}{2}} e^{-\frac{y}{2}}}{\Gamma\left(\nu-\alpha+\frac{1}{2}\right)} \int_{0}^{\infty} e^{-y t} t^{\nu-\alpha-\frac{1}{2}}(1+t)^{\nu+\alpha-\frac{1}{2}} d t, \quad(\alpha \in \mathbb{R}, \nu \in \mathbb{C})
$$

is the Whittaker function.

Assume $f$ is normalized so that $A(\infty, 1)=1$. If $n=\operatorname{sgn}(n) p_{1}^{m_{1}} p_{2}^{m_{2}} \cdots p_{r}^{m_{r}}$ for distinct primes $p_{1}, \cdots, p_{r}$ and positive integers $m_{1}, \cdots, m_{r}$, then we have

$$
A(\infty, n)=A(\infty, \operatorname{sgn}(n)) \prod_{i=1}^{r} A\left(\infty, p_{i}^{m_{i}}\right) .
$$

Remark: In general, the coefficients $A(\mathfrak{a}, n)$ depend on the choice of $\gamma_{\mathfrak{a}}$ and not only on the choice of $\mathfrak{a}$.

\section{WhitTAKER FUNCTIONS FOR THE ADELIC LIFT OF A NEWFORM}

Let $\mathbb{A}$ be the ring of adeles over $\mathbb{Q}$. A place of $\mathbb{A}$ is defined to be either a rational prime or $\infty$. For a finite prime $p$ and for $x \in \mathbb{Q}_{p}$ let

$$
\{x\}:= \begin{cases}\sum_{i=-k}^{-1} a_{i} p^{i}, & \text { if } x=\sum_{i=-k}^{\infty} a_{i} p^{i} \in \mathbb{Q}_{p}, \text { with } k>0,0 \leq a_{i} \leq p-1 ; \\ 0, & \text { otherwise. }\end{cases}
$$

We now define an additive character $e_{v}$ at each place $v$. If $x \in \mathbb{Q}_{v}$, we let

$$
e_{v}(x):= \begin{cases}e^{2 \pi i x}, & \text { if } v=\infty \\ e^{-2 \pi i\{x\}}, & \text { if } v<\infty\end{cases}
$$

Furthermore, for $x=\left\{x_{v}\right\}_{v} \in \mathbb{A}$, define a global additive character for $\mathbb{A}$ as

$$
e(x):=\prod_{\substack{v \leq \infty \\ 4}} e_{v}\left(x_{v}\right)
$$


Let $\chi$ be a Dirichlet character modulo $N$. As is well known, there is a character $\chi_{\text {idelic }}: \mathbb{Q}^{\times} \backslash \mathbb{A}^{\times} \rightarrow \mathbb{C}^{\times}$ associated to $\chi$ which we shall refer to as the idelic lift of $\chi$. For the convenience of the reader we repeat the definition

Definition 7. (Idelic lift of a Dirichlet character) Let $\chi$ be a Dirichlet character of conductor $p^{f}$ where $p^{f}$ is a fixed prime power. We define the idelic lift of $\chi$ to be the unitary Hecke character $\chi_{\text {idelic }}: \mathbb{Q}^{\times} \backslash \mathbb{A}^{\times} \rightarrow$ $\mathbb{C}^{\times}$defined as

$$
\chi_{\text {idelic }}(g):=\chi_{\infty}\left(g_{\infty}\right) \cdot \chi_{2}\left(g_{2}\right) \cdot \chi_{3}\left(g_{3}\right) \cdots, \quad\left(g=\left\{g_{\infty}, g_{2}, g_{3}, \ldots\right\} \in \mathbb{A}^{\times}\right),
$$

where

$$
\chi_{\infty}\left(g_{\infty}\right)=\left\{\begin{aligned}
1, & \chi(-1)=1 \\
1, & \chi(-1)=-1, g_{\infty}>0 \\
-1, & \chi(-1)=-1, g_{\infty}<0
\end{aligned}\right.
$$

and where

$$
\chi_{v}\left(g_{v}\right)= \begin{cases}\chi(v)^{m}, & \text { if } g_{v} \in v^{m} \mathbb{Z}_{v}^{\times} \text {and } v \neq p, \\ \chi(j)^{-1}, & \text { if } g_{v} \in p^{k}\left(j+p^{f} \mathbb{Z}_{p}\right) \text { with } j, k \in \mathbb{Z},(j, p)=1 \text { and } v=p .\end{cases}
$$

More generally, every Dirichlet character $\chi$ of conductor $q=\prod_{i=1}^{r} p_{i}^{f_{i}}$, where $p_{1}, p_{2}, \ldots p_{r}$ are distinct primes and $f_{1}, f_{2}, \ldots f_{r} \geq 1$, can be factored as $\chi=\prod_{i=1}^{r} \chi^{(i)}$, where $\chi^{(i)}$ is a Dirichlet character of conductor $p_{i}^{f_{i}}$. It follows that $\chi$ may be lifted to a Hecke character $\chi_{\text {idelic }}$ on $\mathbb{A}^{\times}$where $\chi_{\text {idelic }}=\prod_{i=1}^{r} \chi_{\text {idelic }}^{(i)}$.

For each $p \mid N$, let

$$
I_{p, N}=\left\{\left(\begin{array}{ll}
a & b \\
c & d
\end{array}\right) \in G L\left(2, \mathbb{Z}_{p}\right) \mid c \equiv 0 \quad(\bmod N)\right\} .
$$

Here $c \equiv 0(\bmod N)$ means $c \in N \cdot \mathbb{Z}_{p}$. Then we can define

$$
K_{0}(N):=\left(\prod_{p \mid N} I_{p, N}\right)\left(\prod_{p \nmid N} G L\left(2, \mathbb{Z}_{p}\right)\right) .
$$

For each $p \mid N$, define a character $\widetilde{\chi_{p}}: I_{p, N} \rightarrow \mathbb{C}^{\times}$, where

$$
\widetilde{\chi_{p}}\left(\left(\begin{array}{ll}
a_{p} & b_{p} \\
c_{p} & d_{p}
\end{array}\right)\right):=\chi_{p}\left(d_{p}\right) .
$$

Define a character $\tilde{\chi}_{\text {idelic }}: K_{0}(N) \rightarrow \mathbb{C}^{\times}$such that

$$
\widetilde{\chi}_{\text {idelic }}(k):=\prod_{p \mid N} \widetilde{\chi_{p}}\left(k_{p}\right)
$$

for all $k=\left\{k_{p}\right\}_{p} \in K_{0}(N)$. If $\chi$ is primitive, then the kernel of $\widetilde{\chi}_{\text {idelic }}$ is given by

$$
\operatorname{Ker}\left(\widetilde{\chi}_{\text {idelic }}\right)=\prod_{p \nmid N} G L\left(2, \mathbb{Z}_{p}\right) \cdot \prod_{p \mid N}\left\{\left(\begin{array}{ll}
a & b \\
c & d
\end{array}\right) \in I_{p, N} \mid d \equiv 1\left(\bmod |N|_{p}^{-1}\right)\right\} .
$$

Let $\mathbb{A}_{\text {finite }}$ denote the finite adeles. For $g=\left\{g_{v}\right\}_{v} \in G L(2, \mathbb{A})$ or $G L\left(2, \mathbb{A}_{\text {finite }}\right)$, define $(g)_{v}:=g_{v}$ where $v$ is a place of $\mathbb{Q}$. Then for $v \leq \infty$, we have the inclusion map $i_{v}: G L\left(2, \mathbb{Q}_{v}\right) \rightarrow G L(2, \mathbb{A})$ defined by

$$
\left(i_{v}\left(g_{v}\right)\right)_{w}:=\left\{\begin{array}{cc}
\left(\begin{array}{ll}
1 & 0 \\
0 & 1
\end{array}\right), & \text { if } w \neq v \\
g_{v}, & \text { if } w=v .
\end{array}\right.
$$

We shall define the diagonal embedding map $i_{\text {diag }}: G L(2, \mathbb{Q}) \rightarrow G L\left(2, \mathbb{A}_{\mathbb{Q}}\right)$ by

$$
i_{\text {diag }}(\gamma):=\{\gamma, \gamma, \gamma, \ldots\}, \quad(\forall \gamma \in G L(2, \mathbb{Q})) .
$$


By strong approximation, it follows that for any $g \in G L(2, \mathbb{A})$, there exist $\gamma \in G L(2, \mathbb{Q}), g_{\infty} \in G L(2, \mathbb{R})^{+}$, $k \in K_{0}(N)$ such that

$$
g=i_{\text {diag }}(\gamma) i_{\infty}\left(g_{\infty}\right) i_{\text {finite }}(k)
$$

where $i_{\text {finite }}$ denotes the diagonal embedding of $G L(2, \mathbb{Q})$ into $G L\left(2, \mathbb{A}_{\text {finite }}\right)$, the group of finite adeles.

Let $f$ be a Maass form of weight $k$, type $\nu$, level $N$ and character $\chi$ modulo $N$. By the above decomposition, we may define a function $f_{\text {adelic }}: G L(2, \mathbb{A}) \rightarrow \mathbb{C}$ as

$$
f_{\text {adelic }}(g):=f_{\text {adelic }}\left(i_{\text {diag }}(\gamma) i_{\infty}\left(g_{\infty}\right) i_{\text {finite }}(k)\right):=\left(\left.f\right|_{k} g_{\infty}\right)(i) \cdot \tilde{\chi}_{\text {idelic }}(k) .
$$

It follows from (3) that $f_{\text {adelic }}$ is well-defined. Further, $f_{\text {adelic }}$ is an adelic automorphic form with a central character $\chi_{\text {idelic }}$.

The function $f_{\text {adelic }}$ has a Fourier expansion,

$$
f_{\text {adelic }}(g)=\sum_{\alpha \in \mathbb{Q}^{\times}} W_{f}\left(\left(\begin{array}{ll}
\alpha & 0 \\
0 & 1
\end{array}\right) g\right)
$$

where

$$
W_{f}(g):=\int_{\mathbb{Q} \backslash \mathbb{A}} f_{\text {adelic }}\left(\left(\begin{array}{ll}
1 & u \\
0 & 1
\end{array}\right) g\right) e(-u) d u
$$

for all $g \in G L(2, \mathbb{A})$ (cf. [15], proof of Lemma 11.1.3). The function $W_{f}(g)$ is called a global Whittaker function for $f_{\text {adelic }}$.

Theorem 9. Let $f: \Gamma_{0}(N) \backslash \mathfrak{h} \rightarrow \mathbb{C}$ be a Maass form of weight $k$, type $\nu$, level $N$, and character $\chi(\bmod N)$. Let $S$ be a set of representatives for the $\Gamma_{0}(N)$-equivalence classes of cusps. Then $f$ has the FourierWhittaker expansion at every cusp as in Proposition [6. By the Iwasawa decomposition, every $g \in G L(2, \mathbb{A})$ has a decomposition

$$
g=\left(\begin{array}{ll}
1 & x \\
0 & 1
\end{array}\right)\left(\begin{array}{ll}
y & 0 \\
0 & 1
\end{array}\right)\left(\begin{array}{ll}
r & 0 \\
0 & r
\end{array}\right) i_{\text {diag }}\left(\left(\begin{array}{ll}
\epsilon & 0 \\
0 & 1
\end{array}\right)\right) k
$$

where $x \in \mathbb{A}, r, y=\left\{y_{v}\right\}_{v} \in \mathbb{A}^{\times}$, with $y_{\infty}>0, k=\left\{k_{v}\right\}_{v} \in K=S O(2, \mathbb{R}) \prod_{p<\infty} G L\left(2, \mathbb{Z}_{p}\right), k_{\infty}=$

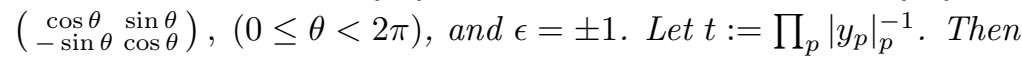

$$
W_{f}(g)=\left\{\begin{array}{cc}
A\left(\mathfrak{a}, \epsilon m_{\mathfrak{a}} t-\mu_{\mathfrak{a}}\right) W_{\frac{\epsilon k}{2}, \nu-\frac{1}{2}}\left(4 \pi y_{\infty}\right) \chi_{\text {idelic }}(r) e(x) e_{\infty}\left(\frac{k \theta}{2 \pi}+t j \epsilon\right) \widetilde{\chi}_{\text {idelic }}\left(k_{0}\right), & \text { if } \epsilon m_{\mathfrak{a}} t-\mu_{\mathfrak{a}} \in \mathbb{Z}, \\
0, & \text { otherwise. }
\end{array}\right.
$$

Here $k_{0} \in K_{0}(N)$, the cusp $\mathfrak{a} \in S$, and an integer $0 \leq j<m_{\mathfrak{a}}$ are uniquely determined by

$$
i_{\text {finite }}\left(\gamma_{\mathfrak{a}}\left(\begin{array}{ll}
1 & j \\
0 & 1
\end{array}\right)\right) \prod_{p \mid N} i_{p}\left(\left(\begin{array}{cc}
t^{-1} y_{p} & 0 \\
0 & 1
\end{array}\right) k_{p}\right)=k_{0} \in K_{0}(N) .
$$

Proof. See [12].

Definition 10. Let $f: \Gamma_{0}(N) \backslash \mathfrak{h} \rightarrow \mathbb{C}$ be a Maass form of weight $k$, type $\nu$, level $N$ and character $\chi$ $(\bmod N)$. For each place $v$, we define a function $W_{f, v}\left(g_{v}\right): G L\left(2, \mathbb{Q}_{v}\right) \rightarrow \mathbb{C}$ as follows.

- $\underline{v=\infty}:$

$$
W_{f, \infty}\left(g_{\infty}\right):=W_{f}\left(i_{\infty}\left(g_{\infty}\right)\right)
$$

- $\underline{v=p<\infty}:$

$$
W_{f, p}\left(g_{p}\right):=\lim _{y_{\infty} \rightarrow 1} \frac{W_{f}\left(i_{\infty}\left(\left(\begin{array}{cc}
y_{\infty} & 0 \\
0 & 1
\end{array}\right)\right) \cdot i_{p}\left(g_{p}\right)\right)}{W_{\frac{k}{2}, \nu-\frac{1}{2}}\left(4 \pi y_{\infty}\right)} .
$$


Remark 11. The purpose of the limit in the definition of $W_{p}$ is to make sure that $W_{p}$ is defined even when $W_{\frac{k}{2}, \nu-\frac{1}{2}}\left(4 \pi y_{\infty}\right)$ happens to vanish at $y_{\infty}=1$. In fact, the ratio $\frac{W_{f}\left(i_{\infty}\left(\left(\begin{array}{cc}y_{\infty} & 0 \\ 0 & 1\end{array}\right)\right) \cdot i_{p}\left(g_{p}\right)\right)}{W_{\frac{k}{2}, \nu-\frac{1}{2}}\left(4 \pi y_{\infty}\right)}$ will turn out to be the same for all $y_{\infty}>0$ such that $W_{\frac{k}{2}, \nu-\frac{1}{2}}\left(4 \pi y_{\infty}\right) \neq 0$.

Corollary 12. Let $f$ be a Maass form of weight $k$, type $\nu$, level $N$ and character $\chi(\bmod N)$. Let $S$ be a set of representatives for the $\Gamma_{0}(N)$-equivalence classes of cusps. Let $\mathfrak{a} \in \mathbb{Q}$ be a cusp for $\Gamma_{0}(N)$. For each integer $n$, let $A(\mathfrak{a}, n)$ be the $n$-th Fourier coefficient of $f$ at the cusp $\mathfrak{a}$ as in Proposition 6 . For every place $v$ of $\mathbb{Q}$ and any $g_{v} \in G L\left(2, \mathbb{Q}_{v}\right)$, we have a decomposition

$$
g_{v}=\left(\begin{array}{cc}
1 & x_{v} \\
0 & 1
\end{array}\right)\left(\begin{array}{cc}
y_{v} & 0 \\
0 & 1
\end{array}\right)\left(\begin{array}{cc}
r_{v} & 0 \\
0 & r_{v}
\end{array}\right) k_{v}
$$

where $x_{v} \in \mathbb{Q}_{v}$ and $y_{v}, r_{v} \in \mathbb{Q}_{v}^{\times}$. If $v=\infty$, then $y_{\infty}>0, \epsilon= \pm 1$ and $k_{\infty} \in\left(\begin{array}{cc}\epsilon & 0 \\ 0 & 1\end{array}\right) S O(2, \mathbb{R})$. If $v$ is finite, then $k_{v} \in G L\left(2, \mathbb{Z}_{v}\right)$.

For each finite prime $p$, fix $y_{p} \in \mathbb{Q}_{p}^{\times}$and $k_{p} \in G L\left(2, \mathbb{Z}_{p}\right)$. Then there exists a cusp $\mathfrak{a}_{p} \in S$, an integer $0 \leq j_{p}<m_{\mathfrak{a}_{p}}$, and $k_{p, 0} \in K_{0}(N)$, which are uniquely determined by $y_{p}$ and $k_{p}$ such that

$$
i_{\text {finite }}\left(\gamma_{\mathfrak{a}_{p}}\left(\begin{array}{cc}
1 & j_{p} \\
0 & 1
\end{array}\right)\right) i_{p}\left(\left(\begin{array}{cc}
\left|y_{p}\right|_{p} y_{p} & 0 \\
0 & 1
\end{array}\right) k_{p}\right)=k_{p, 0} \in K_{0}(N) .
$$

Then by Definition 10, for each place $v$ for $\mathbb{Q}$, we have the following:

- $\underline{v=\infty}$ :

$$
W_{f, \infty}\left(g_{\infty}\right)=A(\infty, \epsilon) W_{\frac{\epsilon k}{2}, \nu-\frac{1}{2}}\left(4 \pi y_{\infty}\right) \chi_{\infty}\left(r_{\infty}\right) e_{\infty}\left(x_{\infty}+\frac{k \theta}{2 \pi}\right)
$$

- $\underline{v=p \nmid N}:$

$$
W_{f, p}\left(g_{p}\right)= \begin{cases}A\left(\infty,\left|y_{p}\right|_{p}^{-1}\right) \chi_{p}\left(r_{p}\right) e_{p}\left(x_{p}\right), & \text { if } y_{p} \in \mathbb{Z}_{p}, \\ 0, & \text { otherwise }\end{cases}
$$

- $\underline{v=p \mid N}:$

$$
W_{f, p}\left(g_{p}\right)=\left\{\begin{array}{l}
A\left(\mathfrak{a}_{p}, m_{\mathfrak{a}_{p}}\left|y_{p}\right|_{p}^{-1}-\mu_{\mathfrak{a}_{p}}\right) \chi_{p}\left(r_{p}\right) e_{p}\left(x_{p}\right) e_{\infty}\left(\left|y_{p}\right|_{p}^{-1} j_{p}\right) \widetilde{\chi}_{\text {idelic }}\left(k_{p, 0}\right), \\
\begin{array}{ll}
0, & \text { otherwise. }
\end{array} m_{\mathfrak{a}_{p}}\left|y_{p}\right|_{p}^{-1}-\mu_{\mathfrak{a}_{p}} \in \mathbb{Z},
\end{array}\right.
$$

Proof. Use Definition 10 and Theorem 9

Remark In Corollary 12, assume that $f$ is a normalized Maass form, i.e., $A(\infty, 1)=1$. Then for $p \nmid N$,

$$
W_{f, p}\left(k_{p}\right)=1, \text { for all } k_{p} \in G L\left(2, \mathbb{Z}_{p}\right),
$$

and for $p \mid N$,

$$
W_{f, p}\left(k_{p}\right)=1, \text { for all } k_{p} \in I_{p, N} \text {. }
$$

The following theorem is well known, but it is usually not presented in the explicit form which we require for our purposes.

Theorem 13. Let $f: \Gamma_{0}(N) \backslash \mathfrak{h} \rightarrow \mathbb{C}$ be a Maass-Hecke newform of weight $k$, type $\nu$, level $N$ and character $\chi(\bmod N)$. If $f$ is normalized, i.e., its first Fourier coefficient at $\infty$ is 1 , then

$$
W_{f}(g)=\prod_{v} W_{f, v}\left(g_{v}\right)
$$


Proof. If $f$ is a Maass-Hecke newform then $f_{\text {adelic }}$ generates an irreducible subspace

$$
V \subset \mathcal{A}_{0}\left(G L(2, \mathbb{Q}) \backslash G L(2, \mathbb{A}), \chi_{\text {idelic }}\right),
$$

and an irreducible automorphic cuspidal representation $(\pi, V)$, under the actions of $G L\left(2, \mathbb{A}_{\text {finite }}\right)$ and $\left(\mathfrak{g}, K_{\infty}\right)$ for $G L(2, \mathbb{R})$, where $\mathfrak{g}=\mathfrak{g l}(2, \mathbb{C})$ and $K_{\infty}=O(2, \mathbb{R})$ (see [4] Theorem 3.6.1). By [7] there are local representations $\left(\pi_{v}, V_{v}\right)$ for $G L\left(2, \mathbb{Q}_{v}\right)$ for each place $v$ of $\mathbb{Q}$ such that

- $\pi_{\infty}$ is an irreducible and admissible $\left(\mathfrak{g}, K_{\infty}\right)$-module;

- $\pi_{v}$ is an irreducible and admissible representation for $G L\left(2, \mathbb{Q}_{v}\right)$ for all finite places $v$. Furthermore, for almost all $v$, we know that $V_{v}$ contains a non-zero $K_{v}$-fixed vector where $K_{v}=G L\left(2, \mathbb{Z}_{v}\right)$, and

$$
(\pi, V) \cong \bigotimes_{v}^{\prime}\left(\pi_{v}, V_{v}\right), \quad \text { (restricted tensor product) }
$$

where the restricted tensor product is taken with respect to some choice of non-zero $K_{v}$-fixed vectors in all but finitely many of the spaces $V_{v}$. (Different choices may give rise to different restricted tensor products, but the representations obtained are all isomorphic to one another and to $(\pi, V)$.)

We shall use the existence and uniqueness of Whittaker models for $(\pi, V)$ and for each of the local representations $\left(\pi_{v}, V_{v}\right)$ (see [4 section 3.5). For each place $v$, let $\mathcal{W}\left(\pi_{v}, e_{v}\right)$ denote the Whittaker space, corresponding to $\left(\pi_{v}, V_{v}\right)$ and the additive character $e_{v}$ introduced at the beginning of this section. For each place $v$ such that $\left(\pi_{v}, V_{v}\right)$ contains a $K_{v}$-fixed vector, the space of $K_{v}$-fixed vectors is one dimensional (see [4] Theorems 2.4.2, 4.6.2), and contains a unique element which takes the value 1 on all of $K_{v}$ (the existence of such an element in the non-archimedean case is proved in 4, Proposition 3.5.2; existence in the Archimedean case can be proved along the same lines but we do not need it here).

Let $\mathcal{W}_{\text {tensor }}$ denote the restricted tensor product of the spaces $\mathcal{W}\left(\pi_{v}, e_{v}\right)$ with respect to the $K_{v}$-fixed vectors which take the value 1 on $K_{v}$. Then pointwise multiplication gives an isomorphism between this space and the unique Whittaker model of the original representation $\pi$.

Indeed, suppose that $g=\left\{g_{v}\right\}_{v} \in G L(2, \mathbb{A})$ and that $\otimes_{v} W_{v}$ is an element of $\mathcal{W}_{\text {tensor }}$, so that for each $v$, the Whittaker function $W_{v}$ is an element of $\mathcal{W}\left(\pi_{v}, e_{v}\right)$, and $W_{v}\left(k_{v}\right)=1$ for all but finitely many $v$ (for all $\left.k_{v} \in K_{v}\right)$. Then the infinite product $\prod_{v} W_{v}\left(g_{v}\right)$ is convergent, because all but finitely many of its terms are 1.

Let $\prod_{v} \mathcal{W}\left(\pi_{v}, e_{v}\right)$ be the space of complex valued functions on $G L(2, \mathbb{A})$ spanned by $\prod_{v} W_{v}\left(g_{v}\right)$ where $W_{v}\left(g_{v}\right) \in \mathcal{W}\left(\pi_{v}, e_{v}\right)$ such that $W_{v}\left(k_{v}\right)=1$ for all $k_{v} \in G L\left(2, \mathbb{Z}_{v}\right)$ for almost all $v<\infty$. (i.e., where $\left.\otimes_{v} W_{v} \in \mathcal{W}_{\text {tensor }} \cdot\right)$

Then

$$
\bigotimes_{v}^{\prime}\left(\pi_{v}, V_{v}\right) \cong \prod_{v} \mathcal{W}\left(\pi_{v}, e_{v}\right)
$$

and $\prod_{v} \mathcal{W}\left(\pi_{v}, e_{v}\right)$ is a global Whittaker function space.

Let

$$
\mathcal{W}(\pi, e):=\left\{W_{\phi}(g):=\int_{\mathbb{Q} \backslash \mathbb{A}} \phi\left(\left(\begin{array}{ll}
1 & u \\
0 & 1
\end{array}\right) g\right) e(-u) d u, \text { for all } g \in G L(2, \mathbb{A}) \mid \phi \in V\right\} .
$$

Then $\mathcal{W}(\pi, e)$ is a Whittaker model isomorphic to $(\pi, V)$. By the global uniqueness of Whittaker models,

$$
(\pi, V) \cong \mathcal{W}(\pi, e)=\prod_{v} \mathcal{W}\left(\pi_{v}, e_{v}\right) \cong \bigotimes_{v}^{\prime}\left(\pi_{v}, V_{v}\right)
$$

It follows from uniqueness of the "local new vector" at each place [5] that the element of the restricted tensor product $\bigotimes_{v} V_{v}$ corresponding to the element $f_{\text {adelic }}$ of $V$ is a pure tensor $\otimes_{v \leq \infty} \xi_{v}$. It follows that $W_{f}(g)=\prod_{v} W_{v}\left(g_{v}\right)$, for some $W_{v}\left(g_{v}\right) \in \mathcal{W}\left(\pi_{v}, e_{v}\right),(v \leq \infty)$ such that $W_{v}\left(k_{v}\right)=1$ for all $k_{v} \in G L\left(2, \mathbb{Z}_{v}\right)$ for almost all $v<\infty$. The functions $W_{v}(v \leq \infty)$ are unique up to scalar. We must show that for each $v \leq \infty$, we can take $W_{v}=W_{f, v}$.

Because $A(\infty, 1) \neq 0$, it follows that $W_{f}\left(i_{\infty}\left(g_{\infty}\right)\right) \neq 0$ for some $g_{\infty} \in G L(2, \mathbb{R})$, and thence that $W_{p}\left(I_{2}\right) \neq$ 0 for each $p<\infty$. (Here, $I_{2}$ is the $2 \times 2$ identity matrix.) We may then normalize $W_{p}$ so that its value at 
$I_{2}$ is 1 , and the equality $W_{\infty}=W_{f, \infty}$ is immediate. Further, $W_{p}\left(g_{p}\right)=W_{f}\left(i_{\infty}\left(g_{\infty}\right) i_{p}\left(g_{p}\right)\right) / W_{f}\left(i_{\infty}\left(g_{\infty}\right)\right)$, for all $g_{p} \in G L\left(2, \mathbb{Q}_{p}\right)$, and any $g_{\infty} \in G L(2, \mathbb{R})$ such that $W_{f}\left(i_{\infty}\left(g_{\infty}\right)\right) \neq 0$. From this it is immediate that $W_{\infty}=W_{f, \infty}$.

Theorem 13 is not "sharp" in the sense that its conclusion is satisfied by the adelic lifts of many forms which are not new, and by many adelic automorphic forms which are not adelic lifts. Combining a careful analysis of the proof of theorem [13 with the strong multiplicity one theorem (lemma 3.1 of [19]) permits us to sharpen the result.

Remarks 14. (1) Clearly the adelic lift $f_{\text {adelic }}$ can be defined for a classical Maass form which is not new. It follows from the strong multiplicity one theorem that the adelic lift generates an irreducible representation of $G L(2, \mathbb{A})$ if an only if $f$ is a linear combination of oldforms which are all obtained from the same newform.

(2) If $\phi$ is any adelic cusp form $G L(2, \mathbb{A}) \rightarrow \mathbb{C}$, then one may consider the corresponding Whittaker function

$$
W_{\phi}(g):=\int_{\mathbb{Q} \backslash \mathbb{A}} f_{\text {adelic }}\left(\left(\begin{array}{ll}
1 & u \\
0 & 1
\end{array}\right) g\right) e(-u) d u,
$$

and (again by strong multiplicity one) the following conditions are equivalent:

- There exist local Whittaker functions $W_{v} \in \mathcal{W}\left(\pi_{v}, e_{v}\right)$ for $v \leq \infty$ such that $W_{\phi}=\prod_{v} W_{v}$.

- The automorphic form $\phi$ generates an irreducible cuspidal automorphic representation $(\pi, V)$ of $G L(2, \mathbb{A})$, and corresponds to a pure tensor under the isomorphism $(\pi, V) \rightarrow \otimes_{v}^{\prime}\left(\pi_{v}, V_{v}\right)$.

(3) The formulae

$$
W_{\infty}\left(g_{\infty}\right)=W\left(i_{\infty}\left(g_{\infty}\right)\right), \quad W_{p}\left(g_{p}\right)=\lim _{y_{\infty} \rightarrow 1} \frac{W\left(i_{\infty}\left(\left(\begin{array}{cc}
y_{\infty} & 0 \\
0 & 1
\end{array}\right) i_{p}\left(g_{p}\right)\right)\right.}{W\left(i_{\infty}\left(\left(\begin{array}{cc}
y_{\infty} & 0 \\
0 & 1
\end{array}\right)\right)\right.},
$$

are valid whenever $W_{p}\left(I_{2}\right) \neq 0$ for all $p<\infty$, or, equivalently, whenever there exists $g_{\infty} \in G L(2, \mathbb{R})$ such that $W\left(i_{\infty}\left(g_{\infty}\right)\right) \neq 0$.

\section{A Classical Consequence of the tensor Product theorem}

The following theorem may be viewed as a translation of the tensor product theorem into a statement about Fourier coefficients of Maass forms. It provides a partial answer to Stark's question.

Theorem 15. Fix a positive integer $N=q_{1}^{e_{1}} \cdots q_{h}^{e_{h}}$ where $q_{1}^{e_{1}}, \cdots, q_{h}^{e_{h}}$ are powers of distinct primes. Let $S$ be a set of inequivalent cusps for $\Gamma_{0}(N)$. Fix an integer $k \geq 1$ and let $f$ be a normalized Maass-Hecke newform of weight $k$, type $\nu$, level $N$ and character $\chi=\prod_{1 \leq i \leq h} \chi_{i}$, where $\chi_{i}$ is a Dirichlet character $\left(\bmod q_{i}^{e_{i}}\right)$ for $1 \leq i \leq h$. Fix one cusp $\mathfrak{a} \in S$ and let $M$ be a positive integer such that

$$
\begin{aligned}
\epsilon M+\mu_{\mathfrak{a}}=\epsilon m_{\mathfrak{a}} p_{1}^{m_{1}} \cdots & p_{n}^{m_{n}} \cdot q_{1}^{m_{1}^{\prime}} \cdots q_{h}^{m_{h}^{\prime}}, \\
& \left(\text { for } m_{i}, m_{j}^{\prime} \in \mathbb{Z}, m_{i}>0, \text { with } 1 \leq i \leq n, 1 \leq j \leq h\right),
\end{aligned}
$$

where $p_{1}, \ldots, p_{n}$ are distinct primes which do not divide $N$.

Then for each $i=1, \ldots, h$, there exist a unique cusp $\mathfrak{b}_{i} \in S$ and a unique integer $1 \leq j_{i}<m_{\mathfrak{b}_{i}}$ such that

$$
\begin{gathered}
\gamma_{\mathfrak{b}_{i}}\left(\begin{array}{cc}
1 & j_{i} \\
0 & 1
\end{array}\right)=:\left(\begin{array}{ll}
a_{i} & b_{i} \\
c_{i} & d_{i}
\end{array}\right), \\
\gamma_{\mathfrak{b}_{i}}\left(\begin{array}{cc}
1 & j_{i} \\
0 & 1
\end{array}\right)\left(\begin{array}{cc}
\epsilon M_{i} & 0 \\
0 & 1
\end{array}\right) \gamma_{\mathfrak{a}}^{-1}=\left(\begin{array}{ll}
a_{q_{i}} & b_{q_{i}} \\
c_{q_{i}} & d_{q_{i}}
\end{array}\right), \quad\left(\text { for } M_{i}:=\frac{\epsilon M+\mu_{\mathfrak{a}}}{\epsilon m_{\mathfrak{a}} q_{i}^{m_{i}^{\prime}}} \in \mathbb{Q}\right),
\end{gathered}
$$

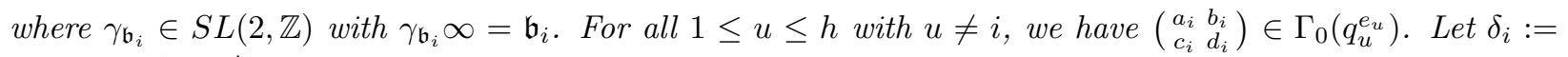
$\prod_{u \neq i} q_{i}^{\max \left(0,-m_{u}^{\prime}\right)}$ and $\delta_{i}^{\prime}$ be an integer such that $\delta_{i}^{\prime} \delta_{i} \equiv 1\left(\bmod q_{i}^{e_{i}}\right)$. Then we have $\delta_{i} a_{q_{i}}, \delta_{i} b_{q_{i}}, \delta_{i} c_{q_{i}}, \delta_{i} d_{q_{i}} \in$ $\mathbb{Z}$ and $\delta_{i} c_{q_{i}} \equiv 0\left(\bmod q_{i}^{e_{i}}\right)$. 
Let $A(\mathfrak{a}, \epsilon M)$ denote the $\epsilon M^{\text {th }}$ Fourier coefficient of $f$ as in Proposition 6 . Then we have

$$
\begin{aligned}
A(\mathfrak{a}, \epsilon M) & =A(\infty, \epsilon) \prod_{i=1}^{n} A\left(\infty, p_{i}^{m_{i}}\right) \\
\cdot & \prod_{i=1}^{h}\left(A\left(\mathfrak{b}_{i}, m_{\mathfrak{b}_{i}} q_{i}^{m_{i}^{\prime}}-\mu_{\mathfrak{b}_{i}}\right) e_{\infty}\left(q_{i}^{m_{i}^{\prime}} j_{i}\right)\left(\prod_{u \neq i, u=1}^{h} \chi_{u}\left(d_{i}\right)^{-1}\right) \chi_{i}\left(\delta_{i} \delta_{i}^{\prime} d_{q_{i}}\right)^{-1}\right)
\end{aligned}
$$

if $m_{\mathfrak{b}_{i}} q_{i}^{m_{i}^{\prime}}-\mu_{\mathfrak{b}_{i}} \in \mathbb{Z}$ for all $i=1, \ldots, h$. Otherwise $A(\mathfrak{a}, \epsilon M)=0$.

Proof. Fix one cusp $\mathfrak{a} \in S$. Then there exists $\gamma_{\mathfrak{a}} \in S L(2, \mathbb{Z})$ such that $\gamma_{\mathfrak{a}} \infty=\mathfrak{a}$. For $y=\left\{y_{v}\right\}_{v} \in \mathbb{A}^{\times}$, $y_{\infty}=1$, let $t=\prod_{p}\left|y_{p}\right|_{p}^{-1}$ and for each $q \mid N$, let

$$
k_{q}:=\left(\begin{array}{cc}
t y_{q}^{-1} & 0 \\
0 & 1
\end{array}\right) \gamma_{\mathfrak{a}}^{-1} .
$$

Then $k_{q} \in G L\left(2, \mathbb{Z}_{q}\right)$ for every $q \mid N$. Let $\epsilon= \pm 1$ and take

$$
g=\left(\begin{array}{ll}
y & 0 \\
0 & 1
\end{array}\right) i_{\text {diag }}\left(\left(\begin{array}{ll}
\epsilon & 0 \\
0 & 1
\end{array}\right)\right) \prod_{q \mid N} i_{q}\left(k_{q}\right) \in G L(2, \mathbb{A}) .
$$

Then

$$
i_{\text {finite }}\left(\gamma_{\mathfrak{a}}\right) \prod_{q \mid N} i_{q}\left(\left(\begin{array}{cc}
t^{-1} y_{q} & 0 \\
0 & 1
\end{array}\right) k_{q}\right) \in K_{0}(N)
$$

since

$$
\gamma_{\mathfrak{a}}\left(\begin{array}{cc}
t^{-1} y_{q} & 0 \\
0 & 1
\end{array}\right) k_{q}=\left(\begin{array}{ll}
1 & 0 \\
0 & 1
\end{array}\right) \in I_{q, N} \text { for each } q \mid N
$$

and $\gamma_{\mathfrak{a}} \in G L\left(2, \mathbb{Z}_{p}\right)$ for any prime $p$.

By Theorem 13, we know that

$$
\begin{aligned}
W_{f} & \left(\left(\begin{array}{ll}
y & 0 \\
0 & 1
\end{array}\right) i_{\text {diag }}\left(\left(\begin{array}{cc}
\epsilon & 0 \\
0 & 1
\end{array}\right)\right) \prod_{q \mid N} i_{q}\left(k_{q}\right)\right) \\
= & W_{f, \infty}\left(\left(\begin{array}{ll}
\epsilon & 0 \\
0 & 1
\end{array}\right)\right)\left(\prod_{p \nmid N} W_{f, p}\left(\left(\begin{array}{cc}
y_{p} & 0 \\
0 & 1
\end{array}\right)\left(\begin{array}{ll}
\epsilon & 0 \\
0 & 1
\end{array}\right)\right)\right) \\
& \cdot\left(\prod_{q \mid N} W_{f, q}\left(\left(\begin{array}{cc}
y_{q} & 0 \\
0 & 1
\end{array}\right)\left(\begin{array}{ll}
\epsilon & 0 \\
0 & 1
\end{array}\right) k_{q}\right)\right) .
\end{aligned}
$$

Then by Corollary 12 for each fixed prime $q \mid N$, there exists a cusp $\mathfrak{b}_{q} \in S$ and an integer $0 \leq j_{q}<m_{\mathfrak{b}_{q}}$ which are uniquely determined by

$$
i_{\text {finite }}\left(\gamma_{\mathfrak{b}_{q}}\left(\begin{array}{cc}
1 & j_{q} \\
0 & 1
\end{array}\right)\right) i_{q}\left(\left(\begin{array}{cc}
\left|y_{q}\right|_{q} y_{q} & 0 \\
0 & 1
\end{array}\right)\left(\begin{array}{cc}
\epsilon & 0 \\
0 & 1
\end{array}\right) k_{q}\right)=: k_{q, 0} \in K_{0}(N)
$$

This is equivalent to

$$
\left(k_{q, 0}\right)_{q}=\gamma_{\mathfrak{b}_{q}}\left(\begin{array}{cc}
1 & j_{q} \\
0 & 1
\end{array}\right)\left(\begin{array}{cc}
\epsilon t\left|y_{q}\right|_{q} & 0 \\
0 & 1
\end{array}\right) \gamma_{\mathfrak{a}}^{-1} \in I_{q, N}
$$

for each prime $q^{\prime} \mid N$ and $q^{\prime} \neq q$,

$$
\left(k_{q, 0}\right)_{q^{\prime}}=\gamma_{\mathfrak{b}_{q}}\left(\begin{array}{cc}
1 & j_{q} \\
0 & 1
\end{array}\right) \in I_{q^{\prime}, N}
$$

and for each prime $p \nmid N$,

$$
\left(k_{q, 0}\right)_{p}=\gamma_{\mathfrak{b}_{q}}\left(\begin{array}{cc}
1 & j_{q} \\
0 & 1
\end{array}\right) \in G L\left(2, \mathbb{Z}_{p}\right) .
$$


It follows form Corollary 12, that

$$
\begin{aligned}
& W_{f}\left(\left(\begin{array}{ll}
y & 0 \\
0 & 1
\end{array}\right) i_{\text {diag }}\left(\left(\begin{array}{ll}
\epsilon & 0 \\
0 & 1
\end{array}\right)\right) \prod_{q \mid N} i_{q}\left(k_{q}\right)\right)=A\left(\mathfrak{a}, \epsilon m_{\mathfrak{a}} t-\mu_{\mathfrak{a}}\right) W_{\frac{\epsilon k}{2}, \nu-\frac{1}{2}}(4 \pi) \\
& =\left\{\begin{array}{c}
A(\infty, \epsilon) W_{\frac{\epsilon k}{2}, \nu-\frac{1}{2}}(4 \pi)\left(\prod_{q \mid N} A\left(\mathfrak{b}_{q}, m_{\mathfrak{b}_{q}}\left|y_{q}\right|_{q}^{-1}-\mu_{\mathfrak{b}_{q}}\right) e_{\infty}\left(\left|y_{q}\right|_{q}^{-1} j_{q}\right) \widetilde{\chi}_{\text {idelic }}\left(k_{q, 0}\right)\right) \\
\prod_{p \nmid N} A\left(\infty,\left|y_{p}\right|_{p}^{-1}\right), \\
\text { if } \epsilon m_{\mathfrak{a}} t-\mu_{\mathfrak{a}} \text { and } m_{\mathfrak{b}_{q}}\left|y_{q}\right|_{q}^{-1}-\mu_{\mathfrak{b}_{q}} \in \mathbb{Z} \text { for all } q \mid N ; \\
0, \quad \text { otherwise. }
\end{array}\right.
\end{aligned}
$$

Therefore,

$$
\begin{aligned}
& A\left(\mathfrak{a}, \epsilon m_{\mathfrak{a}} t-\mu_{\mathfrak{a}}\right) \\
& =\left\{\begin{array}{c}
A(\infty, \epsilon)\left(\prod_{q \mid N} A\left(\mathfrak{b}_{q}, m_{\mathfrak{b}_{q}}\left|y_{q}\right|_{q}^{-1}-\mu_{\mathfrak{b}_{q}}\right) e_{\infty}\left(\left|y_{q}\right|_{q}^{-1} j_{q}\right) \tilde{\chi}_{\text {idelic }}\left(k_{q, 0}\right)\right) \\
\prod_{p \nmid N} A\left(\infty,\left|y_{p}\right|_{p}^{-1}\right), \\
\quad \text { if } \epsilon m_{\mathfrak{a}} t-\mu_{\mathfrak{a}}, m_{\mathfrak{b}_{q}}\left|y_{q}\right|_{q}^{-1}-\mu_{\mathfrak{b}_{q}} \in \mathbb{Z} \text { for all } q \mid N, \\
0, \quad \text { otherwise. }
\end{array}\right.
\end{aligned}
$$

Now let $\epsilon m_{\mathfrak{a}} t-\mu_{\mathfrak{a}}=\epsilon M \in \mathbb{Z}$. Then $\epsilon m_{\mathfrak{a}} t=\epsilon M+\mu_{\mathfrak{a}}$. Since $t=\prod_{p}\left|y_{p}\right|_{p}^{-1}$,

$$
\epsilon M+\mu_{\mathfrak{a}}=\epsilon m_{\mathfrak{a}} p_{1}^{m_{1}} \cdots p_{n}^{m_{n}} q_{1}^{m_{1}^{\prime}} \cdots q_{h}^{m_{h}^{\prime}}
$$

for distinct primes $p_{1}, \ldots, p_{n}$ (different from $q_{1}, \ldots, q_{h}$ ), and $m_{1}, \ldots, m_{n}, m_{1}^{\prime}, \ldots, m_{h}^{\prime} \in \mathbb{Z}$. This means that for each prime $p$, we take

$$
y_{p}= \begin{cases}p_{i}^{m_{i}}, & \text { if } p=p_{i} \text { for some } i=1, \ldots, n, \\ q_{i}^{m_{i}^{\prime}}, & \text { if } p=q_{i} \text { for some } i=1, \ldots, h, \\ 1, & \text { otherwise. }\end{cases}
$$

It follows that if $m_{i}<0$, then $A\left(\infty, p_{i}^{m_{i}}\right)=0$. So assume that $m_{1}, \ldots, m_{n}>0$. Then for each $i=1, \ldots, h$, we use the uniqueness property above for choosing $\mathfrak{b}_{i}$ and integers $1 \leq j_{i}<m_{\mathfrak{b}_{i}}$.

For later purposes, we now describe a convenient set of representatives for the equivalence classes of cusps in the case when $N=q^{e}$ is a prime power.

Lemma 16. If $q$ is a prime, and e a positive integer, then the set

$$
\{0, \infty\} \cup\left\{\frac{1}{c_{1} q^{l}} \mid \begin{array}{c}
1 \leq l<e, \operatorname{gcd}\left(c_{1}, q\right)=1, \\
1 \leq c_{1}<\min \left(q^{l}, q^{e-l}\right),
\end{array}\right\}
$$

is a set of representatives for the $\Gamma_{0}\left(q^{e}\right)$-equivalence classes of cusps.

Proof. It is well known and easily verified that the group $S L(2, \mathbb{Z})$ permutes the set of cusps transitively. It follows that $\Gamma_{0}\left(q^{e}\right)$-equivalence classes of cusps are naturally identified with double $\operatorname{cosets} \Gamma_{0}\left(q^{e}\right) \backslash S L(2, \mathbb{Z}) / \widetilde{\Gamma}_{\mathfrak{a}}$, where $\tilde{\Gamma}_{\mathfrak{a}}$ denotes the stabilizer in $S L(2, \mathbb{Z})$ of any fixed cusp $\mathfrak{a}$. It is convenient to employ this identification with $\mathfrak{a}=\infty$. As remarked above, the stabilizer $\widetilde{\Gamma}_{\infty}=\Gamma_{\infty}$ is independent of $N$ and given explicitly by

$$
\Gamma_{\infty}=\left\{\left(\begin{array}{cc}
\epsilon & n \\
0 & \epsilon
\end{array}\right) \mid \epsilon \in\{ \pm 1\}, n \in \mathbb{Z}\right\} .
$$

Now, it follows easily from the definition of $\Gamma_{0}\left(q^{e}\right)$ that $\Gamma_{0}\left(q^{e}\right) \backslash S L(2, \mathbb{Z})$ is naturally identified with

$$
B^{1}\left(\mathbb{Z} / q^{e} \mathbb{Z}\right) \backslash S L\left(2, \mathbb{Z} / q^{e} \mathbb{Z}\right),
$$


where $B^{1}(R)$ denotes the group of upper triangular matrices with entries in the ring $R$ and determinant equal to 1 . The projective line $\mathbb{P}^{1}\left(\mathbb{Z} / q^{e} \mathbb{Z}\right)$ is given by

$$
\left\{\left(x_{0}, x_{1}\right) \in\left(\mathbb{Z} / q^{e} \mathbb{Z}\right)^{2} \mid\left\langle x_{0}, x_{1}\right\rangle=\mathbb{Z} / q^{e} \mathbb{Z}\right\} / \sim .
$$

Here, $\left\langle x_{0}, x_{1}\right\rangle$ denotes the ideal generated by $x_{0}$ and $x_{1}$, and $\sim$ denotes the equivalence relation given by

$$
\left(x_{0}, x_{1}\right) \sim\left(x_{0}^{\prime}, x_{1}^{\prime}\right) \Longleftrightarrow\left(x_{0}^{\prime}, x_{1}^{\prime}\right)=\left(\lambda x_{0}, \lambda x_{1}\right) \text {, some } \lambda \in\left(\mathbb{Z} / q^{e} \mathbb{Z}\right)^{\times} .
$$

We write $\left[x_{0}: x_{1}\right]$ for the equivalence class of $\left(x_{0}, x_{1}\right)$. The group $S L\left(2, \mathbb{Z} / q^{e} \mathbb{Z}\right)$, has a natural right action on $\mathbb{P}^{1}\left(\mathbb{Z} / q^{e} \mathbb{Z}\right)$ given by

$$
\left[x_{0}: x_{1}\right] \cdot\left(\begin{array}{ll}
a & b \\
c & d
\end{array}\right)=\left[a x_{0}+c x_{1}: b x_{0}+d x_{1}\right], \quad\left[x_{0}: x_{1}\right] \in \mathbb{P}^{1}\left(\mathbb{Z} / q^{e} \mathbb{Z}\right),\left(\begin{array}{ll}
a & b \\
c & d
\end{array}\right) \in S L\left(2, \mathbb{Z} / q^{e} \mathbb{Z}\right) .
$$

Clearly, the stabilizer of $[0: 1]$ is $B^{1}\left(\mathbb{Z} / q^{e} \mathbb{Z}\right)$. Thus $\mathbb{P}^{1}\left(\mathbb{Z} / q^{e} \mathbb{Z}\right)$ may be identified with the coset space $\Gamma_{0}\left(q^{e}\right) \backslash S L(2, \mathbb{Z})$. It follows that $\Gamma_{0}\left(q^{e}\right) \backslash S L(2, \mathbb{Z}) / \Gamma_{\infty}$ is in one-to-one correspondence with orbits for the action of $\Gamma_{\infty}$ on $\mathbb{P}^{1}\left(\mathbb{Z} / q^{e} \mathbb{Z}\right)$ via inclusion into $S L(2, \mathbb{Z})$ and then projection to $S L\left(2, \mathbb{Z} / q^{e} \mathbb{Z}\right)$. Note that the coset in $\Gamma_{0}\left(q^{e}\right) \backslash S L(2, \mathbb{Z})$ which corresponds to the element $\left[x_{0}: x_{1}\right] \in \mathbb{P}^{1}\left(\mathbb{Z} / q^{e} \mathbb{Z}\right)$ consists of all matrices $\left(\begin{array}{ll}a & b \\ c & d\end{array}\right)$ such that $(c, d) \equiv\left(\lambda x_{0}, \lambda x_{1}\right)\left(\bmod q^{e}\right)$ for some $\lambda \in\left(\mathbb{Z} / q^{e} \mathbb{Z}\right)^{\times}$.

It is clear that

$$
\mathbb{P}^{1}\left(\mathbb{Z} / q^{e} \mathbb{Z}\right)=\left\{\left[1: x_{1}\right] \mid x_{1} \in \mathbb{Z} / q^{e} \mathbb{Z}\right\} \cup\left\{\left[x_{0}: 1\right] \mid x_{0} \in \mathbb{Z} / q^{e} \mathbb{Z}-\left(\mathbb{Z} / q^{e} \mathbb{Z}\right)^{\times}\right\},
$$

and that the action of $\Gamma_{\infty}$ permutes the elements of $\left\{\left[1: x_{1}\right] \mid x_{1} \in \mathbb{Z} / q^{e} \mathbb{Z}\right\}$ transitively. It follows that the $\Gamma_{0}\left(q^{e}\right)$-cosets corresponding to these elements comprise a single double coset in $\Gamma_{0}\left(q^{e}\right) \backslash S L(2, \mathbb{Z}) / \Gamma_{\infty}$ which is represented by $\left(\begin{array}{cc}0 & -1 \\ 1 & 0\end{array}\right)$. This matrix maps $\infty$ the the cusp 0 .

We study the action of $\Gamma_{\infty}$ on $\left\{\left[x_{0}: 1\right] \mid x_{0} \in \mathbb{Z} / q^{e} \mathbb{Z}-\left(\mathbb{Z} / q^{e} \mathbb{Z}\right)^{\times}\right\}$. Writing $x_{0}=q^{l} c_{1}$ with $1 \leq l \leq e, 1 \leq$ $c_{1}<q^{e-l}$ and $\operatorname{gcd}\left(c_{1}, q\right)=1$, we compute

$$
\left[q^{l} c_{1}: 1\right]\left(\begin{array}{cc}
1 & n \\
0 & 1
\end{array}\right)=\left[q^{l} c_{1}: q^{l} c_{1} n+1\right]=\left[q^{l} c_{1} \overline{\left(q^{l} c_{1} n+1\right)}: 1\right]
$$

where $\bar{a}$ denotes $a^{-1}$ modulo $q^{e-l}$. From this we see at once that each part of the partition

$$
\bigcup_{l=1}^{e}\left\{\left[q^{l} c_{1}: 1\right] \mid\left(c_{1}, q\right)=1,1 \leq c_{1}<q^{e-l}\right\}
$$

is preserved by the action of $\Gamma_{\infty}$, and that $\Gamma_{\infty}$ acts trivially on

$$
\left\{\left[q^{l} c_{1}: 1\right] \mid\left(c_{1}, q\right)=1,1 \leq c_{1}<q^{e-l}\right\}
$$

whenever $e-l \leq l$, for in this case $\overline{\left(q^{l} c_{1} n+1\right)} \equiv 1\left(\bmod q^{e-l}\right)$, whence $\left[q^{l} c_{1} \overline{\left(q^{l} c_{1} n+1\right)}: 1\right]=\left[q^{l} c_{1}: 1\right]$. When $l=e,\left\{\left[q^{l} c_{1}: 1\right] \mid\left(c_{1}, q\right)=1,1 \leq c_{1}<q^{e-l}\right\}=[0: 1]$, which corresponds to the element of $\Gamma_{0}\left(q^{e}\right) \backslash S L(2, \mathbb{Z})$ represented by the identity matrix. The corresponding cusp is $\infty$. For other values of $l \geq \frac{e}{2}$, we have shown that for each $c_{1}$ such that $1 \leq c_{1}<q^{e-l}$ and $\operatorname{gcd}\left(c_{1}, q\right)=1$, the coset in $\Gamma_{0}\left(q^{e}\right) \backslash S L(2, \mathbb{Z})$ corresponding to $\left[q^{l} c_{1}: 1\right]$, is in fact a double coset $\Gamma_{0}\left(q^{e}\right) \backslash S L(2, \mathbb{Z}) / \Gamma_{\infty}$. This coset is represented by the matrix $\left(\begin{array}{cc}1 & 0 \\ q^{l} c_{1} & 1\end{array}\right)$ which maps $\infty$ to $\frac{1}{q^{l} c_{1}}$.

When $e-l>l$, the action of $\left(\begin{array}{cc}1 & n \\ 0 & 1\end{array}\right)$ on $\left\{\left[q^{l} c_{1}: 1\right] \mid\left(c_{1}, q\right)=1,1 \leq c_{1}<q^{e-l}\right\}$ factors through the function $n \mapsto \overline{\left(q^{l} c_{1} n+1\right)}$. This maps $\mathbb{Z}$ into the group $U_{l}$ of units in $\mathbb{Z} / q^{e} \mathbb{Z}$ which are equivalent to 1 modulo $q^{l}$. It is easy to see that this function is surjective. More precisely $n \mapsto c_{1} n$ is a bijection $\mathbb{Z} / q^{e-l} \mathbb{Z} \rightarrow \mathbb{Z} / q^{e-l} \mathbb{Z}$, while $m \mapsto 1+q^{l} m$ is a bijection $\mathbb{Z} / q^{e-l} \mathbb{Z} \rightarrow U_{l}$, and ${ }^{-}$is a bijection $U_{l} \rightarrow U_{l}$.

Thus we are reduced to studying the action of $U_{l}$ on $\left(\mathbb{Z} / q^{e-l} \mathbb{Z}\right)^{\times}$. Clearly $c_{1} u \equiv c_{1}\left(\bmod q^{l}\right)$ for all $c_{1} \in\left(\mathbb{Z} / q^{e-l} \mathbb{Z}\right)^{\times}$, and $u \in U_{l}$. Equally clearly, if $c_{1}, c_{1}^{\prime} \in\left(\mathbb{Z} / q^{e-l} \mathbb{Z}\right)^{\times}$, and $c_{1} \equiv c_{1}^{\prime}\left(\bmod q^{l}\right)$ then $c_{1}^{\prime} \overline{c_{1}} \in U_{l}$. It follows that the orbits for the action of $U_{l}$ on $\left(\mathbb{Z} / q^{e-l} \mathbb{Z}\right)^{\times}$, are precisely the residue classes modulo $q^{l}$. This completes the proof. 
For a prime $q$ and positive integer $e$, fix $N=q^{e}$. From Lemma 16, we can take the complete set of inequivalent cusps for $\Gamma_{0}\left(q^{e}\right)$ as

$$
S=\{0, \infty\} \cup\left\{\frac{1}{c_{1} q^{l}} \begin{array}{c}
1 \leq l<e, \operatorname{gcd}\left(c_{1}, q\right)=1, \\
1 \leq c_{1}<\min \left(q^{l}, q^{e-l}\right),
\end{array}\right\} .
$$

For each cusp $\mathfrak{a} \in S$, we have the following.

$$
\begin{cases}\mathfrak{a}=0: & \gamma_{\mathfrak{a}}=\left(\begin{array}{cc}
0 & -1 \\
1 & 0
\end{array}\right), \sigma_{\mathfrak{a}}=\left(\begin{array}{cc}
0 & -1 \\
1 & 0
\end{array}\right)\left(\begin{array}{cc}
q^{\frac{e}{2}} & 0 \\
0 & q^{-\frac{e}{2}}
\end{array}\right), g_{\mathfrak{a}}=\left(\begin{array}{cc}
1 & 0 \\
q^{e} & 1
\end{array}\right), m_{\mathfrak{a}}=q^{e}, \\
\mathfrak{a}=\frac{1}{c q^{l}}, l \leq \frac{e}{2}: & \gamma_{\mathfrak{a}}=\left(\begin{array}{cc}
1 & 0 \\
q^{l} c & 1
\end{array}\right), \sigma_{\mathfrak{a}}=\left(\begin{array}{cc}
1 & 0 \\
q^{l} c & 1
\end{array}\right)\left(\begin{array}{cc}
q^{\frac{e}{2}-l} & 0 \\
0 & q^{l-\frac{e}{2}}
\end{array}\right), \\
g_{\mathfrak{a}} & =\left(\begin{array}{cc}
1-q^{e-l} c & q^{e-2 l} \\
-q^{e} c^{2} & 1+q^{e-l} c
\end{array}\right), m_{\mathfrak{a}}=q^{e-2 l}, \\
\mathfrak{a}=\frac{1}{c q^{l}}, l>\frac{e}{2}: & \gamma_{\mathfrak{a}}=\left(\begin{array}{cc}
1 & 0 \\
q^{l} c & 1
\end{array}\right), \sigma_{\mathfrak{a}}=\left(\begin{array}{cc}
1 & 0 \\
q^{l} c & 1
\end{array}\right), \\
g_{\mathfrak{a}} & =\left(\begin{array}{cc}
1-q^{l} c & 1, \\
-q^{2 l} c^{2} & 1+q^{l} c
\end{array}\right), m_{\mathfrak{a}}=1 .\end{cases}
$$

From the above table, we can easily see that $\mu_{0}=\mu_{\infty}=0$ since $\widetilde{\chi}\left(g_{0}\right)=\widetilde{\chi}\left(g_{\infty}\right)=1$. If $\mathfrak{a} \neq 0, \infty$, then $\mathfrak{a}=\frac{1}{c q^{l}}$, with $1 \leq c<\min \left(q^{l}, q^{e-l}\right)$ and $(c, q)=1$ and

$$
\tilde{\chi}\left(g_{\mathfrak{a}}\right)=\chi\left(1+c \cdot \max \left(q^{l}, q^{e-l}\right)\right)=\chi\left(1+\max \left(q^{l}, q^{e-l}\right)\right)^{c}=e^{2 \pi i \mu_{\mathfrak{a}}} .
$$

So $\widetilde{\chi}\left(g_{\mathfrak{a}}\right)^{\min \left(q^{l}, q^{e-l}\right)}=1$.

Lemma 18. Fix an integer $e \geq 1$. Let $\chi$ be a Dirichlet character of prime power level $N=q^{e}$. Let $\chi_{\text {trivial }}$ be the trivial character modulo $q^{e}$. Let $\chi_{0}$ be a primitive Dirichlet character of prime power level $N_{0}=q^{e_{0}}$ (with $\left.0 \leq e_{0} \leq e\right)$ such that $\chi=\chi_{0} \cdot \chi_{\text {trivial }}$. Then the following hold.

- For each integer $1 \leq l<e$ with $\max \left(q^{l}, q^{e-l}\right) \geq q^{e_{0}}$, and any cusp $\mathfrak{a}=\frac{1}{c q^{l}}$ with $1 \leq c<\min \left(q^{l}, q^{e-l}\right)$ and $(c, q)=1$, the cusp parameter $\mu_{\mathfrak{a}}$ is zero.

- For each integer $1 \leq l<e$ with $\max \left(q^{l}, q^{e-l}\right)<q^{e_{0}}$, there exists a cusp $\mathfrak{a}_{0}=\frac{1}{c_{0} q^{l}}$ with cusp parameter $\mu_{\mathfrak{a}_{0}}=\min \left(q^{e_{0}-l}, q^{e_{0}-e+l}\right)^{-1}$. Here $1 \leq c_{0}<\min \left(q^{e_{0}-l}, q^{e_{0}-e+l}\right)$ and $\left(c_{0}, q\right)=1$. Then

$$
\widetilde{\chi}\left(g_{\mathfrak{a}_{0}}\right)=e^{2 \pi i \min \left(q^{e_{0}-l}, q^{e_{0}-e+l}\right)^{-1}}
$$

and for any cusp $\mathfrak{a}=\frac{1}{c q^{l}}$ with $1 \leq c<\min \left(q^{l}, q^{e-l}\right)$ and $(c, q)=1$, there exists a unique integer $1 \leq r<\min \left(q^{e_{0}-l}, q^{e_{0}-e+l}\right)$ with $(r, q)=1$ such that

$$
c \equiv r c_{0}\left(\bmod \min \left(q^{e_{0}-l}, q^{e_{0}-e+l}\right)\right) \text { and } \mu_{\mathfrak{a}}=r \mu_{\mathfrak{a}_{0}} .
$$

Proof. For any integer $m$ with $1 \leq m<e$, let $U_{m}=\left\{a \in\left(\mathbb{Z} / q^{e} \mathbb{Z}\right)^{\times} \mid a \equiv 1(\bmod q)^{m}\right\}$. This is a subgroup. In fact, it is the kernel of the natural projection from $\left(\mathbb{Z} / q^{e} \mathbb{Z}\right)^{\times}$to $\left(\mathbb{Z} / q^{m} \mathbb{Z}\right)^{\times}$. The integer $e_{0}$ is the smallest integer such that $\chi$ factors through this projection. Thus the restriction $\left.\chi\right|_{U_{m}}$ of $\chi$ to $U_{m}$ is trivial iff $m \geq e_{0}$.

Use the set of representatives for cusps $S$ in (17). As we see from the table above, the lower right entry $d_{\mathfrak{a}}$ of the generator $g_{\mathfrak{a}}$ is an element of $U_{\max l, e-l}$. Since $\mu_{\mathfrak{a}}$ is defined so that $e^{2 \pi i \mu_{\mathfrak{a}}}=\chi\left(d_{\mathfrak{a}}\right)$, we need to study the restriction $\left.\chi\right|_{U_{\max (l, e-l)}}$.

If $e_{0} \leq \max (l, e-l)$, this restriction is trivial and $\mu_{\mathfrak{a}}$ is zero, regardless of $c$. The function $c \mapsto 1+c q^{\max (l, e-l)}$ is an isomorphism $\mathbb{Z} / q^{\min (l, e-l)} \mathbb{Z} \rightarrow U_{\max (l, e-l)}$. Composing with $\chi$, we obtain a homomorphism $\varphi$ from $\mathbb{Z} / q^{\min (l, e-l)} \mathbb{Z}$ to $\mathbb{C}$. For any $m$ with $\max (l, e-l) \leq m \leq e$, the preimage of $U_{m}$ in $\mathbb{Z} / q^{\min (l, e-l)} \mathbb{Z}$ is the cyclic subgroup generated by $q^{m-\max (l, e-l)}$, and these are the only subgroups of $\mathbb{Z} / q^{\min (l, e-l)} \mathbb{Z}$. Since the kernel of $\chi$ contains $U_{e_{0}}$, but not $U_{e_{0}-1}$, it follows that the kernel of $\left.\chi\right|_{U_{\max l, e-l}}$ is precisely equal to $U_{e_{0}}$, and that its image is the $\left(q^{e_{0}-\max (l, e-l)}\right)^{\text {th }}$ roots of unity. Furthermore, $\varphi$ factors through the natural projection $\mathbb{Z} / q^{\min (l, e-l)} \mathbb{Z} \rightarrow \mathbb{Z} / q^{e_{0}-\max (l, e-l)}$. For $c_{0}$, we take the least positive element of the residue class which maps to $e^{2 \pi i \min \left(q^{e_{0}-l}, q^{e_{0}-e+l}\right)^{-1}}$. 
The following theorem provides a partial answer to Stark's question in the case of prime power level.

Theorem 19. Let $q^{e}$ be a fixed prime power. Let $f$ be a Hecke-Maass newform of level $q^{e}$, character $\chi$ $\left(\bmod q^{e}\right)$, weight $k$, type $\nu$ for $\Gamma_{0}\left(q^{e}\right)$. Assume $\chi=\chi_{0} \cdot \chi_{\text {trivial }}$ where $\chi_{\text {trivial }}$ is the trivial character modulo $q$ and $\chi_{0}$ is a primitive Dirichlet character of prime power level $q^{e_{0}}$ (with $0 \leq e_{0} \leq e$ ). For $\mathfrak{a} \in S$ and $n \in \mathbb{Z}$, let $A(\mathfrak{a}, n)$ denote the $n^{\text {th }}$ Fourier coefficient of $f$ at the cusp $\mathfrak{a}$ as in Proposition 6 . Assume that $A(\infty, 1)=1$. For any $\mathfrak{a} \in S$ and an arbitrary non-negative integer $M$ let

$$
\epsilon M+\mu_{\mathfrak{a}}=\epsilon m_{\mathfrak{a}} p_{1}^{m_{1}} \cdots p_{n}^{m_{n}} \cdot q^{m}
$$

where $\epsilon= \pm 1, m_{1}, \ldots, m_{n}$ are positive integers, $m \in \mathbb{Z}, p_{1}, \ldots, p_{n}$ are distinct primes different from $q$, and $\mu_{\mathfrak{a}}$ is the cusp parameter given in (5). Set $M_{0}=p_{1}^{m_{1}} \cdots p_{n}^{m_{n}}$. For each cusp $\mathfrak{a}=\frac{1}{c q^{l}} \in S$, there exists a unique cusp $\frac{1}{c^{\prime} q^{l}} \in S$, determined by the conditions

$$
1 \leq c^{\prime} \leq \min \left(q^{e-l}, q^{l}\right), \quad c^{\prime} \in M_{0} \equiv c\left(\bmod \min \left(q^{e-l}, q^{l}\right)\right) .
$$

If $l \leq e / 2$ then there is, in addition, a unique integer $j$ determined by the conditions

$$
0 \leq j<q^{e-2 l}, \quad c c^{\prime} j \equiv\left(c^{\prime} \in M_{0}-c\right) \cdot q^{-l}\left(\bmod q^{e-2 l}\right) .
$$

Let $\alpha \geq 0$ denote the greatest integer such that $q^{\alpha} \mid M$. Then $m, \mu_{\mathfrak{a}}$ and $A(\mathfrak{a}, \epsilon M)$ are given as follows.

$\underline{\mathfrak{a}=\infty}$ : In this case $\mu_{\infty}=0, m=\alpha$, and

$$
A(\infty, \epsilon M)=A(\infty, \epsilon) A\left(\infty, p_{1}^{m_{1}}\right) \cdots A\left(\infty, p_{n}^{m_{n}}\right) A\left(\infty, q^{m}\right) .
$$

$\underline{\bullet} \mathfrak{a}=0:$ In this case $\mu_{0}=0, m=\alpha-e$, and

$$
A(0, \epsilon M)=A\left(\infty, \epsilon M_{0}\right) A\left(0, q^{e+m}\right) \chi\left(\epsilon M_{0}\right)^{-1} .
$$

$\underline{\mathfrak{a}=\frac{1}{c q^{l}}}$, and $\mu_{\mathfrak{a}} \neq 0$ : In this case $e_{0}>\max (l, e-l), m=-e_{0}+l$, and

$$
A\left(\frac{1}{c q^{l}}, \epsilon M\right)=\left\{\begin{array}{c}
A\left(\infty, \epsilon M_{0}\right) A\left(\frac{1}{c^{\prime} q^{l}}, 0\right) e_{\infty}\left(q^{m} \cdot j\right) \chi\left(j c^{\prime} q^{l}+1\right)^{-1}, \\
\quad \text { if } l \leq \frac{e}{2}, \\
A\left(\infty, \epsilon M_{0}\right) A\left(\frac{1}{c^{\prime} q^{l}}, 0\right), \\
\quad \text { if } l>\frac{e}{2},
\end{array}\right.
$$

Furthermore, the cusp parameter of $\frac{1}{c^{\prime} q^{l}} \in S$ is $\min \left(q^{e_{0}-l}, q^{e_{0}-e+l}\right)^{-1}$. (If $e_{0}=e$, i.e., if $\chi$ is primitive, then there is a unique cusp $\mathfrak{a}_{0}=\frac{1}{c_{0} q^{l}} \in S$ having this property, so that $c^{\prime}=c_{0}$, independently of $c, \epsilon$, and $M !)$

$\bullet \mathfrak{a}=\frac{1}{c q^{l}}$, and $\mu_{\mathfrak{a}}=0:$ In this case $m=\alpha-\max (e-2 l, 0)$ and

$$
A\left(\frac{1}{c q^{l}}, \epsilon M\right)=\left\{\begin{aligned}
A\left(\infty, \epsilon M_{0}\right) A\left(\frac{1}{c^{\prime} q^{l}}, q^{e-2 l+m}\right) e_{\infty}\left(q^{m} \cdot j\right) \chi\left(j c^{\prime} q^{l}+1\right)^{-1} \\
\text { if } l \leq \frac{e}{2} \\
A\left(\infty, \epsilon M_{0}\right) A\left(\frac{1}{c^{\prime} q^{l}}, q^{m}\right) \\
\text { if } l>\frac{e}{2}
\end{aligned}\right.
$$

Proof. Fix $\mathfrak{a} \in S$. Let $M$ be a positive integer and $\epsilon= \pm 1$. Write

$$
\epsilon M+\mu_{\mathfrak{a}}=\epsilon m_{\mathfrak{a}} p_{1}^{m_{1}} \cdots p_{n}^{m_{n}} q^{m},
$$

for distinct primes $p_{1}, \cdots, p_{n} \neq q$, and integers $m_{1}, \cdots, m_{n}, m$ with $m_{1}, \cdots, m_{n}>0$. It follows from Lemma 18 and the discussion preceding it that $\mu_{\mathfrak{a}}=0$ except when $\mathfrak{a}=\frac{1}{c q^{l}}$ with $\max (l, e-l)<e_{0}$. Also, in all cases, $m_{\mathfrak{a}}$ is a power of $q$. Thus, when $\mu_{\mathfrak{a}}=0$, the expression

$$
M=p_{1}^{m_{1}} \cdots p_{n}^{m_{n}} \cdot\left(m_{\mathfrak{a}} q^{m}\right)
$$

is the prime factorization of $M$. The expressions for $m$ in terms of $\alpha$ in the various cases now follow easily from the values of $m_{\mathfrak{a}}$ tabulated above. 
When $\mathfrak{a}=\frac{1}{c q^{l}}$ with $\max (l, e-l)<e_{0}$, it follows from Lemma 18 that $\mu_{\mathfrak{a}}$ is a rational number of the form $\frac{r}{\min \left(q^{e_{0}-l}, q^{e_{0}-e+l}\right)}=\frac{r}{q^{e_{0}-\max (l, e-l)}}$ with $\operatorname{gcd}(r, q)=1$. Consequently $\epsilon M+\mu_{\mathfrak{a}}$ is a rational number with the same denominator, and a numerator which is congruent to $r\left(\bmod \min \left(q^{e_{0}-l}, q^{e_{0}-e+l}\right)\right)$. Since in this case $m_{\mathfrak{a}}=q^{\max (l, e-l)-l}$, we obtain $m=-e_{0}+l$.

Let

$$
M_{0}:=\frac{\epsilon M+\mu_{\mathfrak{a}}}{\epsilon m_{\mathfrak{a}} q^{m}}=p_{1}^{m_{1}} \cdots p_{n}^{m_{n}} .
$$

Then by Theorem [15, there exists a unique cusp $\mathfrak{b} \in S$ and a unique integer $1 \leq j<m_{\mathfrak{b}}$ such that

$$
\begin{gathered}
\gamma_{\mathfrak{b}}\left(\begin{array}{ll}
1 & j \\
0 & 1
\end{array}\right)=:\left(\begin{array}{ll}
a & b \\
c & d
\end{array}\right) \in S L(2, \mathbb{Z}), \\
\gamma_{\mathfrak{b}}\left(\begin{array}{ll}
1 & j \\
0 & 1
\end{array}\right)\left(\begin{array}{cc}
\epsilon M_{0} & 0 \\
0 & 1
\end{array}\right) \gamma_{\mathfrak{a}}^{-1}=:\left(\begin{array}{ll}
a_{q} & b_{q} \\
c_{q} & d_{q}
\end{array}\right),
\end{gathered}
$$

where $c_{q} \equiv 0\left(\bmod q^{e}\right)$. Then

$$
A(\mathfrak{a}, \epsilon M)=A(\infty, \epsilon) \prod_{i=1}^{n} A\left(\infty, p_{i}^{m_{i}}\right)\left(A\left(\mathfrak{b}, m_{\mathfrak{b}} q^{m}-\mu_{\mathfrak{b}}\right) e_{\infty}\left(q^{m} \cdot j\right) \chi\left(d_{q}\right)^{-1}\right) .
$$

(we shall show that $m_{\mathfrak{b}} q^{m}-\mu_{\mathfrak{b}}$ is always integral).

(1) If $\mathfrak{a}=\infty$, then $\mu_{\mathfrak{a}}=0$ and $m_{\mathfrak{a}}=1$. Since $\gamma_{\infty}\left(\begin{array}{cc}\epsilon M_{0} & 0 \\ 0 & 1\end{array}\right) \gamma_{\infty}^{-1}=\left(\begin{array}{cc}\epsilon M_{0} & 0 \\ 0 & 1\end{array}\right) \in I_{q, N}$. So $\mathfrak{b}=\infty$. Since $m=\alpha>0$ and $\mu_{\mathfrak{b}}=0$, it follows at once that $m_{\mathfrak{b}} q^{m}-\mu_{\mathfrak{b}} \in \mathbb{Z}$. Furthermore,

$$
A(\infty, \epsilon M)=A(\infty, \epsilon) \prod_{i=1}^{n} A\left(\infty, p_{i}^{m_{i}}\right) \cdot A\left(\infty, q^{m}\right) .
$$

(2) If $\mathfrak{a}=0$, then $\mu_{\mathfrak{a}}=0$ and $m_{\mathfrak{a}}=q^{e}$. Then $\gamma_{0}\left(\begin{array}{cc}\epsilon M_{0} & 0 \\ 0 & 1\end{array}\right) \gamma_{0}^{-1}=\left(\begin{array}{cc}1 & 0 \\ 0 & \epsilon M_{0}\end{array}\right) \in I_{q, N}$. Therefore, $\mathfrak{b}=0$ and $j=0$. Once again $\mu_{\mathfrak{b}}=0$. Furthermore, $m_{\mathfrak{b}} q^{m}=q^{\alpha} \in \mathbb{Z}$. Finally,

$$
A(0, \epsilon M)=A(\infty, \epsilon) \prod_{i=1}^{n} A\left(\infty, p_{i}^{m_{i}}\right) \cdot A\left(0, q^{e+m}\right) \chi\left(\epsilon M_{0}\right)^{-1}
$$

If $\mathfrak{a} \neq 0, \infty$ then $\mathfrak{a}=\frac{1}{c q^{l}}$ for some fixed integers $1 \leq l<e$ and $1 \leq c<\min \left(q^{l}, q^{e-l}\right)$ with $(c, q)=1$. Also, $m_{\mathfrak{a}}=\max \left(q^{e-2 l}, 1\right)$. Let us explicitly determine $\mathfrak{b}$ in this case. First, assume $l \geq \frac{e}{2}$. Consider the computation

$$
\begin{aligned}
\gamma_{\mathfrak{b}}\left(\begin{array}{cc}
\epsilon M_{0} & 0 \\
0 & 1
\end{array}\right) \gamma_{\mathfrak{a}}^{-1} & =\left(\begin{array}{cc}
1 & 0 \\
c^{\prime} q^{l} & 1
\end{array}\right)\left(\begin{array}{cc}
\epsilon M_{0} & \\
0 & 1
\end{array}\right)\left(\begin{array}{cc}
1 & 0 \\
-c q^{l} & 1
\end{array}\right) \\
& =\left(\begin{array}{cc}
\epsilon M_{0} & 0 \\
q^{l}\left(c^{\prime} \epsilon M_{0}-c\right) & 1
\end{array}\right) .
\end{aligned}
$$

It is clear that the matrix on the right-hand side is an element of $I_{q, N}$ if and only if $c \equiv c^{\prime} \in M_{0}\left(\bmod q^{e-l}\right)$. Thus $\mathfrak{b}=\frac{1}{c^{\prime} q^{l}}$ for this particular value of $c^{\prime}$. Referring to the table above, we see that $m_{\mathfrak{b}}=m_{\mathfrak{a}}=1$, and $d_{\mathfrak{a}} \equiv d_{\mathfrak{b}}^{\epsilon M_{0}}\left(\bmod q^{e}\right)$, whence $\epsilon M_{0} \mu_{\mathfrak{b}}-\mu_{\mathfrak{a}} \in \mathbb{Z}$.

Now assume $l<\frac{e}{2}$. Consider the computation

$$
\begin{aligned}
& \gamma_{\mathfrak{b}}\left(\begin{array}{ll}
1 & j \\
0 & 1
\end{array}\right)\left(\begin{array}{cc}
\epsilon M_{0} & 0 \\
0 & 1
\end{array}\right) \gamma_{\mathfrak{a}}^{-1}=\left(\begin{array}{cc}
1 & 0 \\
c^{\prime} q^{l} & 1
\end{array}\right)\left(\begin{array}{ll}
1 & j \\
0 & 1
\end{array}\right)\left(\begin{array}{cc}
\epsilon M_{0} & 0 \\
0 & 1
\end{array}\right)\left(\begin{array}{cc}
1 & 0 \\
-c q^{l} & 1
\end{array}\right) \\
& =\left(\begin{array}{cc}
\epsilon M_{0}-j c q^{l} & j \\
q^{l}\left(c^{\prime} \epsilon M_{0}-j c^{\prime} c q^{l}-c\right) & j c^{\prime} q^{l}+1
\end{array}\right) .
\end{aligned}
$$

It is clear that the matrix on the right-hand side is an element of $I_{q, N}$ if and only if $c^{\prime}$ and $j$ are such that $c \equiv c^{\prime} \epsilon M_{0}\left(\bmod q^{e-l}\right)$ and $j c^{\prime} c \equiv\left(\frac{c^{\prime} \epsilon M_{0}-c}{q^{l}}\right)(\bmod q)^{e-2 l}$. This shows that $\mathfrak{b}=\frac{1}{c^{\prime} q^{l}}$, where $c^{\prime}$ is the unique solution to $c \equiv c^{\prime} \in M_{0}\left(\bmod q^{e-l}\right)$ in the range $1 \leq c^{\prime}<q^{l}$. It follows at once that $m_{\mathfrak{b}}=m_{\mathfrak{a}}=q^{e-2 l}$, and that $\epsilon M_{0} \mu_{\mathfrak{b}}-\mu_{\mathfrak{a}} \in \mathbb{Z}$. 
(3) If $\mu_{\mathfrak{a}} \neq 0$, then by Lemma 18, $\max \left(q^{l}, q^{e-l}\right)<q^{e_{0}}$ and

$$
\mu_{\mathfrak{a}}=\frac{r}{\min \left(q^{e_{0}-l}, q^{e_{0}-e+l}\right)},
$$

for some integer $r$ with $1 \leq r<\min \left(q^{e_{0}-l}, q^{e_{0}-e+l}\right)$. Similiarly

$$
\mu_{\mathfrak{b}}=\frac{r^{\prime}}{\min \left(q^{e_{0}-l}, q^{e_{0}-e+l}\right)},
$$

for some integer $r^{\prime}$ with $1 \leq r^{\prime}<\min \left(q^{e_{0}-l}, q^{e_{0}-e+l}\right)$. Since

$$
\epsilon M+\frac{r}{\min \left(q^{e_{0}-l}, q^{e_{0}-e+l}\right)}=\epsilon m_{\mathfrak{a}} p_{1}^{m_{1}} \cdots p_{n}^{m_{n}} q^{m}=\epsilon M_{0} \max \left(q^{e-2 l}, 1\right) \cdot q^{m}
$$

it follows that $\epsilon M \min \left(q^{e_{0}-l}, q^{e_{0}-e+l}\right)+r=\epsilon M_{0} q^{m+e_{0}-l}$. This implies that $m=-e_{0}+l$ and $\epsilon M_{0} \equiv r$ $\left(\bmod \min \left(q^{e_{0}-l}, q^{e_{0}-e+l}\right)\right)$. Since $\epsilon M_{0} \mu_{\mathfrak{b}}-\mu_{\mathfrak{a}} \in \mathbb{Z}$, we deduce that $\epsilon M_{0} r^{\prime} \equiv r\left(\bmod \min \left(q^{e_{0}-l}, q^{e_{0}-e+l}\right)\right)$, and hence that $r^{\prime}=1$. It follows that $\mu_{\mathfrak{b}}=\min \left(q^{e_{0}-l}, q^{e_{0}-e+l}\right)^{-1}=m_{\mathfrak{b}} q^{m}$.

- $l \leq e-l$ and $\mu_{\mathfrak{a}}=r \mu_{\mathfrak{a}_{0}} \neq 0$ : In this case it follows from (21) and the definitions of $c^{\prime}$ and $j$ that

$$
A\left(\frac{1}{c q^{l}}, \epsilon M\right)=A(\infty, \epsilon) \prod_{i=1}^{n} A\left(\infty, p_{i}^{m_{i}}\right)\left(A\left(\frac{1}{c^{\prime} q^{l}}, 0\right) e_{\infty}\left(q^{-e_{0}+l} \cdot j\right) \chi\left(j c^{\prime} q^{l}+1\right)^{-1}\right)
$$

where $c^{\prime}$ and $j$ are determined by $c$ and $\epsilon M_{0}$ as above.

- $l>e-l$ and $\mu_{\mathfrak{a}}=r \mu_{\mathfrak{a}_{0}} \neq 0$ : In this case it follows from (201) and the definition of $c^{\prime}$ that

$$
A\left(\frac{1}{c q^{l}}, \epsilon M\right)=A(\infty, \epsilon) \prod_{i=1}^{n} A\left(\infty, p_{i}^{m_{i}}\right) A\left(\frac{1}{c^{\prime} q^{l}}, 0\right),
$$

where $c^{\prime}$ is determined by $c$ and $\epsilon M_{0}$ as above.

(4) For a fixed integer $1 \leq l<e$, take an integer $1 \leq c<\min \left(q^{l}, q^{e-l}\right)$ and $(c, q)=1$. Let $\mathfrak{a}=\frac{1}{c q^{l}}$. As shown above, $\mathfrak{b}=\frac{1}{c^{\prime} q}$, where $c^{\prime}$ is the unique solution to

$$
1 \leq c^{\prime}<\min \left(q^{l}, q^{e-l}\right) \quad c^{\prime} \epsilon M_{0}-c \equiv 0 \quad\left(\bmod \min \left(q^{l}, q^{e-l}\right)\right) .
$$

Assume that $\mu_{\mathfrak{a}}=0$. Then $\mu_{\mathfrak{b}}=0$ by Lemma 18. Furthermore $m_{\mathfrak{b}}=m_{\mathfrak{a}}=q^{\max (e-2 l, 0)}$. It follows that $q^{m} m_{\mathfrak{b}}-\mu_{\mathfrak{b}}=q^{\alpha}$ (where $\alpha$ is the highest pwer of $q$ that divides $M$ as before), which is integral.

- $l \leq e-l$ and $\mu_{\mathfrak{a}}=0$ : In this case it follows from (21) and the definitions of $c^{\prime}$ and $j$ that

$$
A\left(\frac{1}{c q^{l}}, \epsilon M\right)=A(\infty, \epsilon) \prod_{i=1}^{n} A\left(\infty, p_{i}^{m_{i}}\right)\left(A\left(\frac{1}{c^{\prime} q^{l}}, q^{e-2 l+m}\right) e_{\infty}\left(q^{m} \cdot j\right) \chi\left(j c^{\prime} q^{l}+1\right)^{-1}\right) .
$$

- $l>e-l$ and $\mu_{\mathfrak{a}}=0$ : In this case it follows from (20) and the definition of $c^{\prime}$ that

$$
A\left(\frac{1}{c q^{l}}, \epsilon M\right)=A(\infty, \epsilon) \prod_{i=1}^{n} A\left(\infty, p_{i}^{m_{i}}\right) \cdot A\left(\frac{1}{c^{\prime} q^{l}}, q^{m}\right)
$$

Remark 22. It is clear from the proofs that theorems 15 and 19 are valid not only for Maass-Hecke newforms, but whenever the factorization $W_{f}=\prod_{v} W_{f, v}$ is valid. 


\section{REMARKS ON CHOICES OF $\mathfrak{a}$ AND $\gamma_{\mathfrak{a}}$}

As remarked in section 1, the Fourier coefficients $A(\mathfrak{a}, n)$ of a Maass form $f$ at a cusp a actually depend on the matrix $\sigma_{\mathfrak{a}}$, or, equivalently, the matrix $\gamma_{\mathfrak{a}}$ used in its definition, and not only on the choice of a. Further, while it is intuitively obvious that when considering Fourier expansions at various cusps, it is sufficient to consider a maximal set of $\Gamma_{0}(N)$-inequivalent cusps, it is also clear that the choice of representative for each $\Gamma_{0}(N)$-equivalence class will influence the precise numbers considered. In this section we make these dependencies completely explicit and then offer some remarks on choice of representatives for $N$ not a prime power.

Because we wish to study the dependence of the Fourier coefficients on the choice of matrix $\gamma_{\mathfrak{a}}$ used to define them, it is necessary to make this dependence explicit. Thus, we write $A\left(\gamma_{\mathfrak{a}}, n\right)$ rather than $A(\mathfrak{a}, n)$.

Lemma 23. Suppose that $\mathfrak{a}$ and $\mathfrak{a}^{\prime}$ are two $\Gamma_{0}(N)$-equivalent cusps, and that $\gamma_{\mathfrak{a}}, \gamma_{\mathfrak{a}^{\prime}}$ are two elements of $S L(2, \mathbb{Z})$ such that $\gamma_{\mathfrak{a}} \infty=\mathfrak{a}$ and $\gamma_{\mathfrak{a}^{\prime}} \infty=\mathfrak{a}^{\prime}$. Let $A\left(\gamma_{\mathfrak{a}}, n\right)$ (resp. $\left.A\left(\gamma_{\mathfrak{a}^{\prime}}, n\right)\right), n \in \mathbb{Z}$ denote the Fourier coefficients of a Maass form $f$ at $\mathfrak{a}$ (resp. $\left.\mathfrak{a}^{\prime}\right)$ defined using an element $\sigma_{\mathfrak{a}}$ (resp. $\sigma_{\mathfrak{a}^{\prime}}$ ) obtained from $\gamma_{\mathfrak{a}}$ (resp. $\gamma_{\mathfrak{a}^{\prime}}$ ) as in section 1. Then

$$
A\left(\gamma_{\mathfrak{a}^{\prime}}, n\right)=\widetilde{\chi}\left(\gamma_{0}\right) \cdot e\left(\left(n+\mu_{\mathfrak{a}}\right) \cdot \frac{j}{\mathfrak{m}_{\mathfrak{a}}}\right) \cdot A\left(\gamma_{\mathfrak{a}}, n\right)
$$

where $\gamma_{0} \in \Gamma_{0}(N)$ and $j \in \mathbb{Z}$ with $0 \leq j<m_{\mathfrak{a}}$ are uniquely determined by the condition that

$$
\gamma_{\mathfrak{a}^{\prime}}=\gamma_{0} \cdot \gamma_{\mathfrak{a}} \cdot\left(\begin{array}{ll}
1 & j \\
0 & 1
\end{array}\right)
$$

Proof. This follows easily from the definitions.

We would like to extend the idea for choosing explicit representatives for the equivalence classes of cusps described in Lemma 16. It is not convenient or necessary to make a completely explicit, choice of cusp representatives. It turns out to be sufficient to specify our representatives only modulo a suitable power of each prime dividing $N$.

For the remainder of this section and the next, we shall employ the following notation. We take $S$ to be a finite set of primes, denoting a general element of $S$ by $q$, and a general prime which is not in $S$ by $p$. For each element $q$ of $S$ we fix a strictly positive integer $e_{q}$, and we let $N=\prod_{q \in S} q^{e_{q}}$.

Lemma 24. For each $q \in S$, let $\pi_{q}: \mathbb{Z} / N \mathbb{Z} \rightarrow \mathbb{Z} / q^{e_{q}} \mathbb{Z}$ denote the natural projection. The natural map $\mathbb{Z} / N \mathbb{Z} \rightarrow \prod_{q \in S} \mathbb{Z} / q^{e_{q}} \mathbb{Z}$ given by $n \mapsto\left(\pi_{q}(n)\right)_{q \in S}$ induces a bijection $\mathbb{P}^{1}(\mathbb{Z} / N \mathbb{Z}) \rightarrow \prod_{q \in S} \mathbb{P}^{1}\left(\mathbb{Z} / q^{e_{q}} \mathbb{Z}\right)$. Furthermore, two elements of $\mathbb{P}^{1}(\mathbb{Z} / N \mathbb{Z})$ are in the same $\Gamma_{\infty}$-orbit if and only if their images in $\mathbb{P}^{1}\left(\mathbb{Z} / q^{e_{q}} \mathbb{Z}\right)$ are in the same $\Gamma_{\infty}$-orbit for all $q \in S$.

Proof. The Chinese remainder theorem states that the natural map $\mathbb{Z} / N \mathbb{Z} \rightarrow \prod_{q \in S} \mathbb{Z} / q^{e_{q}} \mathbb{Z}$ is a ring isomorphism. It follows easily that gives a bijection

$$
\left\{\left(x_{0}, x_{1}\right) \in(\mathbb{Z} / N \mathbb{Z})^{2} \mid\left\langle x_{0}, x_{1}\right\rangle=\mathbb{Z} / N \mathbb{Z}\right\} \rightarrow \prod_{q \in S}\left\{\left(x_{0}, x_{1}\right) \in\left(\mathbb{Z} / q^{e_{q}} \mathbb{Z}\right)^{2} \mid\left\langle x_{0}, x_{1}\right\rangle=\mathbb{Z} / q^{e_{q}} \mathbb{Z}\right\} .
$$

Furthermore, if $\left(x_{0}^{\prime}, x_{1}^{\prime}\right)=\left(\lambda x_{0}, \lambda x_{1}\right)$, then $\left(\pi_{q}\left(x_{0}^{\prime}\right), \pi_{q}\left(x_{1}^{\prime}\right)\right)=\left(\pi_{q}(\lambda) \pi_{q}\left(x_{0}\right), \pi_{q}(\lambda) \pi_{q}\left(x_{1}\right)\right)$ for each $q \in S$. Finally, suppose for each $q \in S$ there exists $\lambda_{q}$ such that $\left(\pi_{q}\left(x_{0}^{\prime}\right), \pi_{q}\left(x_{1}^{\prime}\right)\right)=\left(\lambda_{q} \pi_{q}\left(x_{0}\right), \lambda_{q} \pi_{q}\left(x_{1}\right)\right)$. Then it follows that $\left(x_{0}^{\prime}, x_{1}^{\prime}\right)=\left(\lambda x_{0}, \lambda x_{1}\right)$, where $\lambda$ is the unique solution to the system of congruences $\pi_{q}(\lambda)=$ $\lambda_{q} \forall q \in S$. Consequently, we have a well-defined bijection

$$
\mathbb{P}^{1}(\mathbb{Z} / N \mathbb{Z}) \rightarrow \prod_{q \in S} \mathbb{P}^{1}\left(\mathbb{Z} / q^{e_{q}} \mathbb{Z}\right)
$$

In the same manner, we see that $\exists n \in \mathbb{Z} / N \mathbb{Z}$ such that $\left[x_{0}: x_{1}\right]=\left[y_{0}: y_{1}\right]\left(\begin{array}{cc}1 & n \\ 0 & 1\end{array}\right)$ if and only if, for each $q$, $\exists n_{q} \in \mathbb{Z} / q^{e_{q}} \mathbb{Z}$ such that $\left[x_{0}: x_{1}\right] \equiv\left[y_{0}: y_{1}\right]\left(\begin{array}{cc}1 & n_{q} \\ 0 & 1\end{array}\right) \quad\left(\bmod q^{e_{q}}\right)$. 
Corollary 25. Suppose that, for each $q \in S$, a set $\mathcal{C}_{q}$ of representatives for the double cosets $\Gamma_{0}\left(q^{e_{q}}\right) \backslash S L(2, \mathbb{Z}) / \Gamma_{\infty}$ has been chosen. Let $\mathcal{C}$ be a set having the property that, for any element $\left(\gamma_{q}\right)_{q \in S}$ of the Cartesian product $\prod_{q \in S} \mathcal{C}_{q}$ there is a unique element $\gamma \in \mathcal{C}$ such that

$$
\gamma \equiv \gamma_{q}\left(\bmod q^{e_{q}}\right), \quad(\forall q \in S) .
$$

Then $\mathcal{C}$ is a set of representatives for the double cosets $\Gamma_{0}(N) \backslash S L(2, \mathbb{Z}) / \Gamma_{\infty}$.

Remark: A choice of representatives for $\Gamma_{0}(N) \backslash S L(2, \mathbb{Z}) / \Gamma_{\infty}$ is slightly more information than a choice of representatives for the $\Gamma_{0}(N)$-equivalence classes of cusps: it includes also a choice of matrix $\gamma_{\mathfrak{a}}$ for each representative cusp $\mathfrak{a}$.

By Corollary 25. we may fix a set $\mathcal{C}$ of representatives for the double cosets $\Gamma_{0}(N) \backslash S L(2, \mathbb{Z}) / \Gamma_{\infty}$ such that, for each $q \in S$ and each $\gamma \in \mathcal{C}$, the matrix $\Gamma$ is equivalent $\left(\bmod q^{e_{q}}\right)$ to one of the representatives for $\Gamma_{0}\left(q^{e_{q}}\right) \backslash S L(2, \mathbb{Z}) / \Gamma_{\infty}$ fixed in Lemma 16 .

$$
\left\{\left(\begin{array}{ll}
1 & 0 \\
0 & 1
\end{array}\right),\left(\begin{array}{cc}
0 & -1 \\
1 & 0
\end{array}\right)\right\} \cup\left\{\left(\begin{array}{cc}
1 & 0 \\
c_{1} q^{l} & 1
\end{array}\right) \mid \begin{array}{c}
1 \leq l<e, \operatorname{gcd}\left(c_{1}, q\right)=1 \\
1 \leq c_{1}<\min \left(q^{l}, q^{e-l}\right)
\end{array}\right\} .
$$

Such a choice determines a maximal set of inequivalent cusps for $\Gamma_{0}(N)$ and a choice of matrix $\gamma_{\mathfrak{a}}$ for each element $\mathfrak{a}$ of this set. Declaring that we choose our representatives $\mathfrak{a}$ and the corresponding matrices $\gamma_{\mathfrak{a}}$ in this manner does not uniquely determine $\gamma_{\mathfrak{a}}$, but it does uniquely determine the coefficients $A\left(\gamma_{\mathfrak{a}}, n\right)$, for if $\gamma_{\mathfrak{a}}$ and $\gamma_{\mathfrak{a}}^{\prime}$ are equivalent $(\bmod N$ ) then they differ by an element of the principal congruence subgroup $\Gamma(N)$ on the left, and $\Gamma(N)$ is contained in the kernel of the character $\tilde{\chi}$.

Lemma 27. Let $\mathfrak{a}$ be a cusp and $\gamma_{\mathfrak{a}}$ a matrix such that $\gamma_{\mathfrak{a}} \infty=\mathfrak{a}$ and, for each $q \in S, \gamma_{\mathfrak{a}}$ is equivalent $(\bmod q)^{e_{q}}$ to one of the elements of (26). Let $\mathfrak{a}_{q}$ denote the corresponding cusp. That is, $\gamma_{\mathfrak{a}_{q}} \infty=\mathfrak{a}_{q}$ with $\gamma_{\mathfrak{a}_{q}}$ from (26) and $\gamma_{\mathfrak{a}} \equiv \gamma_{\mathfrak{a}_{q}}\left(\bmod q^{e_{q}}\right)$. Let $\mu_{\mathfrak{a}}$ be the cusp parameter of $\mathfrak{a}$, defined using some character $\chi$ $(\bmod N)$. The isomorphism $\mathbb{Z} / N \mathbb{Z} \rightarrow \prod_{q \in S} \mathbb{Z} / q^{e_{q}} \mathbb{Z}$ ensures that $\chi=\prod_{q \in S} \chi_{q}$ for some characters $\left(\chi_{q}\right)_{q \in S}$ with $\chi_{q}\left(\bmod q^{e q}\right)$ for each $q$. For each $q \in S$, let $\mu_{\mathfrak{a}_{q}}$ denote the cusp parameter of $\mathfrak{a}_{q}$ relative to $\chi_{q}$. Then

$$
\begin{gathered}
m_{\mathfrak{a}}=\operatorname{lcm}_{q \in S}\left(m_{\mathfrak{a}_{q}}\right), \\
\mu_{\mathfrak{a}}=\sum_{q \in S} \frac{m_{\mathfrak{a}}}{m_{\mathfrak{a}_{q}}} \mu_{\mathfrak{a}_{q}}-\left\lfloor\sum_{q \in S} \frac{m_{\mathfrak{a}}}{m_{\mathfrak{a}_{q}}} \mu_{\mathfrak{a}_{q}}\right\rfloor \quad \text { (greatest integer function). }
\end{gathered}
$$

Proof. The lower left entry of $\gamma_{\mathfrak{a}} \cdot\left(\begin{array}{ll}1 & j \\ 0 & 1\end{array}\right) \cdot \gamma_{\mathfrak{a}}^{-1}$ is congruent to $0(\bmod N)$ if and only if it is congruent to $0\left(\bmod q^{e_{q}}\right)$ for each $q$. This is the case if and only if $j$ is divisible by $m_{\mathfrak{a}_{q}}$ for each $q$. The first statement follows. We see at once that for each $q \in S$,

$$
\gamma_{\mathfrak{a}} \cdot\left(\begin{array}{cc}
1 & m_{\mathfrak{a}} \\
0 & 1
\end{array}\right) \cdot \gamma_{\mathfrak{a}}^{-1} \equiv \gamma_{\mathfrak{a}_{q}} \cdot\left(\begin{array}{cc}
1 & m_{\mathfrak{a}} \\
0 & 1
\end{array}\right) \cdot \gamma_{\mathfrak{a}_{q}}^{-1}=\gamma_{\mathfrak{a}_{q}} \cdot\left(\begin{array}{cc}
1 & m_{\mathfrak{a}_{q}} \\
0 & 1
\end{array}\right)^{\frac{m_{\mathfrak{a}}}{m_{\mathfrak{a}_{q}}}} \cdot \gamma_{\mathfrak{a}_{q}}^{-1} \quad\left(\bmod q^{e_{q}}\right) .
$$

It follows that $d_{\mathfrak{a}} \equiv d_{\mathfrak{a}_{q}}^{\frac{m_{\mathfrak{a}}}{m_{q}}}\left(\bmod q^{e_{q}}\right)$ for all $q \in S$ and from this the second assertion follows immediately.

The following lemma will be useful later on.

Lemma 28. Let $\mathfrak{a}$ be a cusp and let $(c, d)$ denote the bottom row of $\gamma_{\mathfrak{a}}$. Let a be an integer prime to $N$. Let $\mathfrak{a}^{\prime}$ be the cusp such that $\gamma_{\mathfrak{a}^{\prime}}$ represents the double coset in $\Gamma_{0}(N) \backslash S L(2, \mathbb{Z}) / \Gamma_{\infty}$ corresponding to the $\Gamma_{\infty}$-orbit of $[a c: d]$ in $\mathbb{P}^{1}(\mathbb{Z} / N \mathbb{Z})$. Then $m_{\mathfrak{a}^{\prime}}=m_{\mathfrak{a}}$ and $\mu_{\mathfrak{a}^{\prime}}-a \cdot \mu_{\mathfrak{a}} \in \mathbb{Z}$.

Proof. For each $q$ in $S$, the pair $(c, d)$ is equivalent to $(0,1),(1,0)$ or $\left(c_{1} q^{l}, 1\right)$ modulo $q^{e_{q}}$ where $c_{1}, l$ are subject to the constraints in (26). It follows at once that the bottom row of $\gamma_{\mathfrak{a}^{\prime}}$ is equivalent to $(0,1),(1,0)$ of $\left(c_{1}^{\prime} q^{l}, 1\right)$, respectively, where $c_{1}^{\prime} \equiv a c_{1}\left(\bmod q^{e_{q}-l}\right)$ and $0<c_{1}^{\prime}<q^{\min \left(l, e_{q}-l\right)}$. The value of $m_{\mathfrak{a}_{q}}$ is 1 , except when the bottom row of $\gamma_{\mathfrak{a}}$ is equivalent to $\left(c_{1} q^{l}, 1\right)$, with $l<e-l$, in which case it is $q^{e-2 l}$. It follows easily that $m_{\mathfrak{a}_{q}^{\prime}}=m_{\mathfrak{a}_{q}}$ for each $q$ and thence that $m_{\mathfrak{a}^{\prime}}=m_{\mathfrak{a}}$. Similarly, $d_{\mathfrak{a}_{q}}$ is equal to 1 if $(c, d) \equiv(0,1)$ or $(1,0)$ 
$\left(\bmod q^{e_{q}}\right)$, while if it is congruent to $\left(c_{1} q^{l}, 1\right)$, then $d_{\mathfrak{a}_{q}}=\left(1+c_{1} q^{\max (l, e-l)}\right)$. Clearly, with $c_{1}^{\prime}$ as above, we have

$$
\left(1+c_{1}^{\prime} q^{\max (l, e-l)}\right) \equiv\left(1+a c_{1} q^{\max (l, e-l)}\right) \equiv\left(1+c_{1}^{\prime} q^{\max (l, e-l)}\right)^{a} \quad\left(\bmod q^{e_{q}}\right) .
$$

It follows that $a \mu_{\mathfrak{a}_{q}}-\mu_{\mathfrak{a}_{q}^{\prime}} \in \mathbb{Z}$. The second assertion of this lemma now follows from Lemma 27. because $m_{\mathfrak{a}} / m_{\mathfrak{a}_{q}}$ and $m_{\mathfrak{a}^{\prime}} / m_{\mathfrak{a}_{q}^{\prime}}$ are the same integer.

It is easy to see from the proof of Lemma 28 that the mapping $(a, \mathfrak{a}) \rightarrow \mathfrak{a}^{\prime}$ which is considered in Lemma 28 actually defines an action of $(\mathbb{Z} / N \mathbb{Z})^{\times}$on our set of cusps. Abusing notation we regard it as an "action" of the set of all elements of $\mathbb{Z}$ which are prime to $N$. We shall write $a \cdot \mathfrak{a}$ for the cusp $\mathfrak{a}^{\prime}$ obtained from $\mathfrak{a}$ in this fashion, and $a^{-1} \cdot \mathfrak{a}$ for the unique cusp $\mathfrak{a}^{\prime \prime} \operatorname{such}$ that $a \cdot \mathfrak{a}^{\prime \prime}=\mathfrak{a}$.

\section{On STARK's QUESTION}

In this section we deduce some consequences of theorem 15 which provide a partial answer to the question of Stark posed in the introduction. We first give in theorem 35 a sufficient condition for multiplicative relations at a cusp, and deduce that there are multiplicative relations at every cusp if $N$ is equal to 4 times a squarefree odd number. Next, if $N$ is equal to 8 times a squarefree odd number, then theorem 35 will imply multiplicative relations at all cusps except for those of the form $a / b$ with $2 \| b$. In proposition 42 we consider such cusps in detail.

In order to begin, it is useful to introduce an alternate notation for the Fourier coefficients. Define

$$
B(\mathfrak{a}, \alpha):=\left\{\begin{array}{ll}
A\left(\mathfrak{a}, m_{\mathfrak{a}} \alpha-\mu_{\mathfrak{a}}\right), & \text { if } m_{\mathfrak{a}} \alpha-\mu_{\mathfrak{a}} \in \mathbb{Z}, \\
0, & \text { otherwise, }
\end{array} \quad\left(\alpha \in \mathbb{Q}^{\times}\right) .\right.
$$

When the dependence of $B(\mathfrak{a}, \alpha)$ on the choice of $\gamma_{\mathfrak{a}} \in S L(2, \mathbb{Z})$ is relevant, we shall denote $B(\mathfrak{a}, \alpha)$ by $B\left(\gamma_{\mathfrak{a}}, \alpha\right)$, where the coefficients $A(\mathfrak{a}, \cdot)$ are defined using $\sigma_{\mathfrak{a}}=\gamma_{\mathfrak{a}}\left(\sqrt{m_{\mathfrak{a}}} \sqrt{m_{\mathfrak{a}}}-1\right)$. Note that this defines $B(\gamma, \alpha)$ for any $\gamma \in S L(2, \mathbb{Z})$ and any $\alpha \in \mathbb{Q}^{\times}$, and permits us to consider, where necessary coefficients defined at the same cusp relative to two different elements of $S L(2, \mathbb{Z})$.

Next, we give a formal definition of what it means for a function $\mathbb{Q}^{\times} \rightarrow \mathbb{C}$ to be multiplicative.

Definition 30. Let $h: \mathbb{Q}^{\times} \rightarrow \mathbb{C}$ be a function. Then $h$ is said to be multiplicative if there exist functions $h_{\infty}:\{ \pm 1\} \rightarrow \mathbb{C}$ and $h_{p}: \mathbb{Z} \rightarrow \mathbb{C}$ for each prime $p$ such that $h_{p}(0)=1$ for all but finitely many primes $p$, and

$$
h\left(\epsilon \prod_{p} p^{e_{p}}\right)=h_{\infty}(\epsilon) \cdot \prod_{p} h_{p}\left(e_{p}\right),
$$

for any $\epsilon \in\{ \pm 1\}$ and $e_{p} \in \mathbb{Z}$ with $e_{p}=0$ for all but finitely many primes $p$.

Remark 32. The functions $h_{\infty}, h_{p}$ are not uniquely determined by $h$ : given a collection of functions satisfying (31), one may vary any finite set of them by nonzero scalars, provided the product of all the scalars is 1 . If $h$ is a factorizable function and $h(1)$ is 1 , then one can normalize by requiring that $h_{\infty}(1)=1$ and $h_{p}(0)=1$ for all $p$. Then (31) becomes

$$
h\left(\epsilon \prod_{p} p^{e_{p}}\right)=h(\epsilon) \cdot \prod_{p} h\left(p^{e_{p}}\right) .
$$

Thus (31) is a generalization of (33), which may be applied to functions with vanish at 1.

In this section, we consider the question:

$$
\text { is the function } \alpha \rightarrow B(\gamma, \alpha) \text { multiplicative? }
$$

We remark that by lemma 23, the answer to the question (34) depends only on the cusp a provided that $\mu_{\mathfrak{a}}=0$ and $m_{\mathfrak{a}}=1$, but not in general. 
Theorem 35. Let $\mathfrak{a}=\frac{a}{b}$ be a cusp, with $\operatorname{gcd}(a, b)=1$, and set $M=\operatorname{gcd}(N, b)$. Assume that either $\operatorname{gcd}(M, N / M)=1$, or else $\operatorname{gcd}(M, N / M)=2$, and $2 \| \frac{N}{M}$. Assume further that for each prime $q \mid N$, the matrix $\gamma_{\mathfrak{a}}$ is equivalent to some element of (26) modulo $q^{e}$, where $q^{e} \| N$. Finally, let $f: \mathfrak{h} \rightarrow \mathbb{C}$ be any Maass form for $\Gamma_{0}(N)$ such that $W_{f}(g)=\prod_{v} W_{f, v}\left(g_{v}\right)$ for all $g=\left\{g_{v}\right\} \in G L(2, \mathbb{A})$. Then the Fourier coefficients $B\left(\gamma_{\mathfrak{a}}, \alpha\right)$ of $f$ at $\mathfrak{a}$ (defined relative to $\gamma_{\mathfrak{a}}$ ) are multiplicative.

Proof. For $\gamma \in S L(2, \mathbb{Z})$ and $\alpha \in \mathbb{Q}^{\times}$, let

$$
g(\gamma, \alpha)=i_{\infty}\left(\left(\begin{array}{ll}
\operatorname{sign}(\alpha) & \\
& 1
\end{array}\right)\right) i_{\text {finite }}\left(\left(\begin{array}{ll}
\alpha & \\
& 1
\end{array}\right) \gamma^{-1}\right) .
$$

Then it follows from theorems 9 and 15 that

$$
\begin{aligned}
B(\gamma, \alpha) & =\lim _{y_{\infty} \rightarrow 1} \frac{W_{f}\left(i_{\infty}\left(\left(\begin{array}{ll}
y_{\infty} & \\
& 1
\end{array}\right)\right) \cdot g(\gamma, \alpha)\right)}{W_{\frac{\operatorname{sign}(\alpha) k}{2}, \nu-\frac{1}{2}}\left(4 \pi y_{\infty}\right)} \\
& =A(\infty, \operatorname{sign}(\alpha)) \cdot \prod_{p} W_{f, p}\left(\left(\begin{array}{ll}
\alpha & \\
& 1
\end{array}\right) \gamma^{-1}\right) .
\end{aligned}
$$

Furthermore,

$$
W_{f, p}\left(\left(\begin{array}{ll}
\alpha & \\
& 1
\end{array}\right) \gamma^{-1}\right)= \begin{cases}A\left(\infty,|\alpha|_{p}^{-1}\right), & \text { if }|\alpha|_{p}^{-1} \in \mathbb{Z} \\
0 & , \text { otherwise }\end{cases}
$$

for all $p \nmid N$. Let $q$ be a prime with $q^{e} \| N, e>0$. In order compute $W_{f, q}\left(\left(\begin{array}{ll}\alpha & \\ & 1\end{array}\right) \gamma_{\mathfrak{a}}^{-1}\right)$ one must determine the unique cusp $\mathfrak{b}(\mathfrak{a}, \alpha, q)$, (from our fixed set of representatives for the $\Gamma_{0}(N)$-equivalence classes), integer $0 \leq j(\mathfrak{a}, \alpha, q) \leq m_{\mathfrak{a}}$, and matrix and $k_{0}(\mathfrak{a}, \alpha, q) \in K_{0}(N)$ such that

$$
i_{\text {finite }}\left(\gamma_{\mathfrak{b}(\mathfrak{a}, \alpha, q)}\left(\begin{array}{cc}
1 & j(\mathfrak{a}, \alpha, q) \\
1
\end{array}\right)\right) i_{q}\left(\left(\begin{array}{ll}
\alpha|\alpha|_{q} & \\
& 1
\end{array}\right) \gamma_{\mathfrak{a}}^{-1}\right)=k_{0}(\mathfrak{a}, \alpha, q)
$$

\begin{tabular}{|c|c|c|c|c|}
\hline$\gamma_{\mathfrak{a}}$ & $\gamma_{\mathfrak{b}}$ & 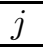 & & $k_{0}$ \\
\hline$(1)$ & 71 & & & $\overline{\alpha \alpha|\alpha|_{q}}$ \\
\hline$\left(\begin{array}{l}1 \\
1\end{array}\right)$ & & 0 & & 1) \\
\hline$\left(\begin{array}{ll}0 & -1\end{array}\right)$ & $\left(\begin{array}{ll}0 & -1\end{array}\right)$ & 0 & & $(1$ \\
\hline$\left(\begin{array}{ll}1 & 0 \\
1 & \end{array}\right)$ & $\begin{array}{l}1 \\
1\end{array}$ & & & $\alpha|\alpha|_{0} \mid{ }^{\mid q}$ \\
\hline$\left(\begin{array}{ll}2^{e-1} & 1\end{array}\right)$ & $\left(\begin{array}{cc}2^{e-1} & 1\end{array}\right)$ & 0 & $\left(2^{e-1}\left(\alpha^{-1}|\alpha|_{2}^{-1}-1\right)\right.$ & 1) $\left(\begin{array}{ll}\left(\begin{array}{ll}|l| l \mid & 1\end{array}\right) & 1\end{array}\right.$ \\
\hline
\end{tabular}

We may assume that the set of representatives for the $\Gamma_{0}(N)$-equivalence classes, and that a matrix $\gamma_{\mathfrak{b}}$ for each representative $\mathfrak{b}$, were chosen as in section 5. Then $\mathfrak{b}(\mathfrak{a}, \alpha, q)$ depends only on the reduction of $\mathfrak{a}$ modulo $q^{e}$. Furthermore, this reduction has to equal $\left(\begin{array}{cc}1 & 0 \\ 0 & 1\end{array}\right)$ or $\left(\begin{array}{cc}0 & -1 \\ 1 & 0\end{array}\right)$, unless $q=2$, in which case $\left(\begin{array}{cc}1 & \\ 2^{e-1} & 1\end{array}\right)$ is also an option. For such matrices the values of $\mathfrak{b}, j$ and $k_{0}$ are as below:

Multiplicativity of $B\left(\gamma_{\mathfrak{a}}, \alpha\right)$ follows easily.

Corollary 39. If $N$ is either squarefree, or equal to 4 times an odd, squarefree number, then $B(\mathfrak{a}, \alpha)$ is multiplicative at every cusp, provided the matrices $\gamma_{\mathfrak{a}}$ are chosen as in section 5 .

Remark 40. If we do not choose the matrices $\gamma_{\mathfrak{a}}$ as in section 5, then the multiplicativity may well be destroyed. For example, suppose $N=q$, a prime, and $\mathfrak{a}=0$. Then $\mu_{0}=0$, and $m_{0}=q$. Thus $B(0, \alpha)$ is zero, unless $\alpha=n / q$ with $n \in \mathbb{Z}$, in which case it is $A(0, n)$. By choosing $\gamma_{0}=\left(\begin{array}{cc}0 & -1 \\ 1 & 0\end{array}\right)$, we obtain the Fricke involution for $\sigma_{0}$, and the Fourier coefficents $A(0, n)$ and $B(0, \alpha)$ are multiplicative exactly as in [1] On the other hand, if we take $\gamma_{0}=\left(\begin{array}{cc}0 & -1 \\ 1 & 1\end{array}\right)$, then, according to Lemma 23, the effect is to multiply $A(0, n)$ by $e_{\infty}\left(\frac{n}{q}\right)$, destroying multiplicativity.

Remark 41. The condition placed on $M$ in theorem 35 can be given more conceptually. What we require is a condition on $\mathfrak{a}$ which will ensure that $\mathfrak{b}$ is independent of $\alpha$, and $j$ is always zero. Next, we examine a case when $\mathfrak{b}$ is independent of $\alpha$, but $j$ is not always zero. 
Proposition 42. Suppose that $N$ is equal to 8 times an odd squarefree number, and that $\mathfrak{a}=a / b$ is a cusp such that $2 \| b$. Assume that $\gamma_{\mathfrak{a}}$ was chosen as in section 可. Then $B(\mathfrak{a}, \alpha)$ is equal to a multiplicative function times the function

$$
\alpha=2^{e} \cdot(1+2 j+4 k) \mapsto e_{\infty}\left(2^{e} \cdot j\right) .
$$

Proof. As in the proof of theorem 35, we use (37). The conditions on $N$ assure that $B(\mathfrak{a}, \alpha, q)$ is independent of $\alpha$ for all $q$, and that $j(\mathfrak{a}, \alpha, q)$ is identically zero for all $q$ except $q=2$. When $q=2$, we must solve

$$
i_{\text {finite }}\left(\gamma_{\mathfrak{b}}\left(\begin{array}{ll}
1 & j \\
& 1
\end{array}\right)\right) i_{2}\left(\left(\begin{array}{ll}
\alpha|\alpha|_{2} & \\
& 1
\end{array}\right) \gamma_{\mathfrak{a}}^{-1}\right) \in K_{0}(N) .
$$

By looking at the condition on $j$ at each prime, we see that $\frac{N}{8} \mid j$, and that

$$
\left(\begin{array}{ll}
1 & \\
2 & 1
\end{array}\right)\left(\begin{array}{ll}
1 & j \\
& 1
\end{array}\right)\left(\begin{array}{ll}
\alpha|\alpha|_{q} & \\
& 1
\end{array}\right)\left(\begin{array}{cc}
1 & \\
-2 & 1
\end{array}\right)=\left(\begin{array}{cc}
\left.\left.\alpha\right|_{\alpha}\right|_{2}-2 j & j \\
2 \alpha|\alpha|_{2}-2(1+2 j) & 1+2 j
\end{array}\right) \in K_{0}(8)
$$

This implies that $j$ satisfies $\alpha|\alpha|_{2} \equiv 1+2 j(\bmod 4)$. Since $\{1,3\}$ is a subgroup of $\mathbb{Z} / 8 \mathbb{Z}^{\times}$, the function

$$
\alpha \mapsto \widetilde{\chi}_{\text {idelic }}\left(\begin{array}{cc}
\alpha|\alpha|_{2}-2 j & j \\
2 \alpha|\alpha|_{2}-2(1+2 j) & 1+2 j
\end{array}\right),
$$

is multiplicative. It follows that $B(\mathfrak{a}, \alpha)$ is a product of functions, all of which are multiplicative except for $\alpha \mapsto e_{\infty}\left(j(\mathfrak{a}, \alpha, 2) \cdot 2^{e}\right)$, which is given by (43).

Certainly, the function (43) is not multiplicative. However, it is still possible for the Fourier coefficients $B(\mathfrak{a}, \alpha)$ to be multiplicative if sufficiently many of them are zero. For example, suppose that $N=8$ and $\chi$ is primitive. Then $\mu_{\mathfrak{a}}=\frac{1}{2}$, and $m_{\mathfrak{a}}=\frac{1}{2}$, from which it follows that $B(\mathfrak{a}, \alpha)=0$ unless $\alpha=\frac{2 n+1}{4}$. The function (43) in this case is 1 if $2 n+1$ is $1 \bmod 4$ and $i$ if it is $3 \bmod 4$. If there exist coprime integers $n_{1}, n_{2}$ with $n_{1} \equiv n_{2} \equiv 3(\bmod 4)$, and $B\left(\mathfrak{a}, \frac{n_{1}}{4}\right) B\left(\mathfrak{a}, \frac{n_{2}}{4}\right) \neq 0$, then it follows that $B(\mathfrak{a}, \alpha)$ is not a multiplicative function. Furthermore, it follows from lemma 23 that $B(\mathfrak{a}, \alpha)$ is not multiplicative for any other choice of $\gamma_{\mathfrak{a}}$ either.

On the other hand, if $A(\infty, n)=0$ whenever $n \equiv 3(\bmod 4)$, then the function (43) can be omitted from the formula for $B\left(\frac{1}{2}, \alpha\right)$ without affecting its value. As a consequence, the Fourier coefficients are multiplicative in this case.

\section{A Classical INTERPRETATION OF THE JACQUET-LANGLANDS CRITERION FOR SUPERCUSPIDALITY}

The following theorem provides a classical criterion for a representation of $G L\left(2, \mathbb{Q}_{p}\right)$ to be supercuspidal.

Theorem 44. Fix a prime $p$, let $V_{p}$ be a complex vector space, and let $\pi_{p}: G L\left(2, \mathbb{Q}_{p}\right) \rightarrow G L\left(V_{p}\right)$. Assume $\left(\pi_{p}, V_{p}\right)$ is an irreducible and admissible representation of $G L\left(2, \mathbb{Q}_{p}\right)$.

Let $f$ be a normalized Maass-Hecke newform of weight $k$, type $\nu$, level $N$, and character $\chi(\bmod N)$ for $\Gamma_{0}(N)$. For each cusp $\mathfrak{a} \in \mathbb{Q} \cup\{\infty\}$ and $n \in \mathbb{Z}$, let $A(\mathfrak{a}, n)$ denote the $n^{\text {th }}$ Fourier coefficient of $f$ at the cusp $\mathfrak{a}$. Let $f_{\text {adelic }}$ be the adelic lift of $f$ as in (8). If $\left(\pi_{p}, V_{p}\right)$ is isomorphic to the local representation factor of the irreducible global automorphic representation of $G L(2, \mathbb{A})$ (which is generated by $\left.f_{\text {adelic }}\right)$, then $\left(\pi_{p}, V_{p}\right)$ is supercuspidal if and only if for each cusp $\mathfrak{a} \in \mathbb{Q} \cup\{\infty\}$ with $\mu_{\mathfrak{a}}=0$, there exists an integer $M_{\mathfrak{a}} \geq 0$, such that

where $m_{\mathfrak{a}}, \mu_{\mathfrak{a}}$ are given by (4), (5), respectively.

$$
A\left(\mathfrak{a}, m_{\mathfrak{a}} p^{m}\right)=0, \quad\left(\text { for all } m \in \mathbb{Z}, m \geq M_{\mathfrak{a}}\right),
$$

Proof. If $p \nmid N$, then $f_{\text {adelic }}$ is fixed by $K_{p}=G L\left(2, \mathbb{Z}_{p}\right)$. It follows that $\left(\pi_{p}, V_{p}\right)$ has a nonzero $K_{p}$-fixed vector, and a nonzero Whittaker model, which forces it to be an irreducible principal series representation (see [4, Theorem 4.6.4). It follows that if $p \nmid N$ then $\left(\pi_{p}, V_{p}\right)$ cannot be a supercuspidal representation. On the other hand, if $p \nmid N$ and $f$ is an eigenfunction of $T_{p}^{\chi}$, then it follows easily that infinitely many of the coefficients $A\left(\infty, p^{m}\right)$ are nonzero. This proves the equivalence in this case, and henceforth we shall assume that $p \mid N$.

Let $W_{f}$ denote the global Whittaker function of $f_{\text {adelic }}$. It follows from Theorem 13, that there exist local Whittaker functions $W_{f, v}$ on $G L\left(2, \mathbb{Q}_{v}\right)$ at each place of $v$ such that

$$
W_{f}(g)=\prod_{v} W_{f, v}\left(g_{v}\right), \quad\left(\forall g=\left\{g_{v}\right\}_{v \leq \infty} \in G L(2, \mathbb{A})\right) .
$$


For a prime $p$, assume that $\left(\pi_{p}, V_{p}\right)$ is isomorphic to a component of the irreducible automorphic representation of $G L(2, \mathbb{A})$ generated by $f_{\text {adelic }}$. As we have noted before (see proof of Theorem 13) there exists a Whittaker space $\mathcal{W}_{p}:=\mathcal{W}\left(\pi_{p}, e_{p}\right)$ associated to $\left(\pi_{p}, V_{p}\right)$ and $W_{f, p} \in \mathcal{W}_{p}$. We also have a corresponding Kirillov space, denoted $\mathcal{K}_{p}$, where

$$
\mathcal{K}_{p}=\left\{W\left(\left(\begin{array}{ll}
y & 0 \\
0 & 1
\end{array}\right)\right) \mid y \in \mathbb{Q}_{p}^{\times}, W \in \mathcal{W}_{p}\right\} .
$$

(The motivation for this name comes from [16], [17].) Define the Schwartz-Bruhat space

$$
S\left(\mathbb{Q}_{p}^{\times}\right):=\left\{\phi: \mathbb{Q}_{p}^{\times} \rightarrow \mathbb{C} \mid \begin{array}{c}
\phi \text { is locally constant, and } \exists N_{\phi}>\epsilon_{\phi}>0 \text { such that } \\
\phi(y)=0 \text { if }|y|_{p}<\epsilon_{\phi} \text { or }|y|_{p}>N_{\phi}
\end{array}\right\} .
$$

It was shown by Jacquet-Langlands that $\left(\pi_{p}, V_{p}\right)$ is supercuspidal if and only if $\mathcal{K}_{p}=S\left(\mathbb{Q}_{p}^{\times}\right)$. (This is proposition 2.16 of [15]. It also appears as theorem 3 of [10]. See [12], for a very elementary treatment.)

Assume that $p \mid N$. For $y \in \mathbb{Q}_{p}^{\times}$, define

$$
\varphi_{p}(y):=W_{f, p}\left(\left(\begin{array}{cc}
y & 0 \\
0 & 1
\end{array}\right)\right) \in \mathcal{K}_{p}
$$

By Corollary 12

$$
\varphi_{p}(y)= \begin{cases}A\left(\infty,|y|_{p}^{-1}\right), & \text { if }|y|_{p}^{-1} \in \mathbb{Z} \\ 0, & \text { otherwise }\end{cases}
$$

Then $\varphi_{p}$ is invariant under the action of

$$
I_{p, N}=\left\{\left(\begin{array}{ll}
a & b \\
c & d
\end{array}\right) \in G L\left(2, \mathbb{Z}_{p}\right) \mid c \in N \mathbb{Z}_{p}\right\}
$$

and $\mathcal{K}_{p}$ is spanned by

$$
\left\{\pi^{\prime}(g) \cdot \varphi_{p} \mid g \in G L\left(2, \mathbb{Q}_{p}\right)\right\} .
$$

Now fix $g=\left(\begin{array}{ll}a & b \\ c & d\end{array}\right) \in G L\left(2, \mathbb{Q}_{p}\right)$. We will compute $\pi^{\prime}(g) \cdot \varphi(y)$ for $y \in \mathbb{Q}_{p}^{\times}$and determine under what conditions this function lies in $S\left(\mathbb{Q}_{p}^{\times}\right)$. There are two different cases that need to be considered.

Case (1) $c=0$ :

$$
\begin{aligned}
\pi^{\prime}\left(\left(\begin{array}{ll}
a & b \\
0 & d
\end{array}\right)\right) \cdot \varphi_{p}(y) & =W_{f, p}\left(\left(\begin{array}{ll}
y & 0 \\
0 & 1
\end{array}\right)\left(\begin{array}{ll}
a & b \\
0 & d
\end{array}\right)\right) \\
& = \begin{cases}\chi_{p}(d) e_{p}\left(b d^{-1} y\right) A\left(\infty,\left|a d^{-1} y\right|_{p}^{-1}\right), & \text { if }\left|a d^{-1} y\right|_{p}^{-1} \in \mathbb{Z}, \\
0, & \text { otherwise. }\end{cases}
\end{aligned}
$$

For fixed $\left(\begin{array}{ll}a & b \\ 0 & d\end{array}\right) \in G L\left(2, \mathbb{Q}_{p}\right)$, the function $\pi^{\prime}\left(\left(\begin{array}{ll}a & b \\ 0 & d\end{array}\right)\right) \cdot \varphi_{p} \in S\left(\mathbb{Q}_{p}^{\times}\right)$if and only if there exists an integer $M \geq 0$ such that $\pi^{\prime}\left(\left(\begin{array}{ll}a & b \\ 0 & d\end{array}\right)\right) \cdot \varphi_{p}(y)=0$ if $y \in p^{M} \mathbb{Z}_{p}$. Since $\chi_{p}(d) \neq 0$ and $e_{p}\left(b d^{-1} y\right) \neq 0$, this function vanishes if and only if $A\left(\infty,\left|a d^{-1} y\right|_{p}^{-1}\right)=0$. It follows that $\pi^{\prime}\left(\left(\begin{array}{ll}a & b \\ 0 & d\end{array}\right)\right) \cdot \varphi_{p} \in S\left(\mathbb{Q}_{p}^{\times}\right)$if and only if there exists a an integer $M_{\infty} \geq 0$ such that $A\left(\infty, p^{m}\right)=0$ whenever $m \geq M_{\infty}$.

$\underline{\text { Case }(2) c \neq 0 \text { : }}$

$$
\left(\begin{array}{ll}
a & b \\
c & d
\end{array}\right)=\left(\begin{array}{ll}
c & 0 \\
0 & c
\end{array}\right)\left(\begin{array}{cc}
c^{-2}(a d-b c) & a c^{-1} \\
0 & 1
\end{array}\right)\left(\begin{array}{cc}
0 & -1 \\
1 & 0
\end{array}\right)\left(\begin{array}{cc}
1 & c^{-1} d \\
0 & 1
\end{array}\right)
$$

Consequently 


$$
\begin{aligned}
& \pi_{p}^{\prime}\left(\left(\begin{array}{ll}
a & b \\
c & d
\end{array}\right)\right) \cdot \varphi_{p}(y) \\
& \quad=\pi_{p}^{\prime}\left(\left(\begin{array}{ll}
c & 0 \\
0 & c
\end{array}\right)\left(\begin{array}{cc}
c^{-2}(a d-b c) & a c^{-1} \\
0 & 1
\end{array}\right)\right) \cdot\left(\pi_{p}^{\prime}\left(\left(\begin{array}{cc}
0 & -1 \\
1 & 0
\end{array}\right)\left(\begin{array}{cc}
1 & c^{-1} d \\
0 & 1
\end{array}\right)\right) \cdot \varphi_{p}\right)(y) \\
& \quad=\chi_{p}(c) e_{p}\left(a c^{-1} y\right)\left(\pi_{p}^{\prime}\left(\left(\begin{array}{cc}
0 & -1 \\
1 & 0
\end{array}\right)\left(\begin{array}{cc}
1 & c^{-1} d \\
0 & 1
\end{array}\right)\right) \cdot \varphi_{p}\right)\left(c^{-2}(a d-b c) y\right) .
\end{aligned}
$$

If $c^{-1} d \in \mathbb{Z}_{p}$ then

$$
\begin{gathered}
\left(\pi_{p}^{\prime}\left(\left(\begin{array}{cc}
0 & -1 \\
1 & 0
\end{array}\right)\left(\begin{array}{cc}
1 & c^{-1} d \\
0 & 1
\end{array}\right)\right) \cdot \varphi_{p}\right)\left(c^{-2}(a d-b c) y\right)=\left(\pi_{p}^{\prime}\left(\left(\begin{array}{cc}
0 & -1 \\
1 & 0
\end{array}\right)\right) \cdot \varphi_{p}\right)\left(c^{-2}(a d-b c) y\right) \\
=W_{f, p}\left(\left(\begin{array}{cc}
c^{-2}(a d-b c) y & 0 \\
0 & 1
\end{array}\right)\left(\begin{array}{cc}
0 & -1 \\
1 & 0
\end{array}\right)\right) .
\end{gathered}
$$

Then by Corollary 12, there exists a cusp $\mathfrak{a} \in \mathbb{Q} \cup\{\infty\}$, an integer $0 \leq j<m_{\mathfrak{a}}$, and $k_{0} \in K_{0}(N)$, which are uniquely determined by $g=\left(\begin{array}{ll}a & b \\ c & d\end{array}\right)$ and $y$ such that

$$
i_{\text {finite }}\left(\gamma_{\mathfrak{a}}\left(\begin{array}{ll}
1 & j \\
0 & 1
\end{array}\right)\right) i_{p}\left(\left(\begin{array}{cc}
\left|c^{-2}(a d-b c) y\right|_{p} c^{-2}(a d-b c) y & 0 \\
0 & 1
\end{array}\right)\left(\begin{array}{cc}
0 & -1 \\
1 & 0
\end{array}\right)\right)=k_{0} \in K_{0}(N) .
$$

Then

$$
\begin{aligned}
& W_{f, p}\left(\left(\begin{array}{cc}
c^{-2}(a d-b c) y & 0 \\
0 & 1
\end{array}\right)\left(\begin{array}{cc}
0 & -1 \\
1 & 0
\end{array}\right)\right) \\
& =\left\{\begin{array}{cc}
A\left(\mathfrak{a}, m_{\mathfrak{a}}\left|c^{-2}(a d-b c) y\right|_{p}^{-1}-\mu_{\mathfrak{a}}\right) e_{\infty}\left(\left|c^{-2}(a d-b c) y\right|_{p}^{-1} j\right) \widetilde{\chi}_{\text {idelic }}\left(k_{0}\right), \\
\text { if } m_{\mathfrak{a}}\left|c^{-2}(a d-b c) y\right|_{p}^{-1}-\mu_{\mathfrak{a}} \in \mathbb{Z}, \\
0, & \text { otherwise. }
\end{array}\right.
\end{aligned}
$$

Therefore, if $c^{-1} d \in \mathbb{Z}_{p}$ then

$$
\begin{aligned}
& \pi_{p}^{\prime}\left(\left(\begin{array}{ll}
a & b \\
c & d
\end{array}\right)\right) \cdot \varphi_{p}(y) \\
& =\left\{\begin{array}{c}
\chi_{p}(c) e_{p}\left(a c^{-1} y\right) A\left(\mathfrak{a}, m_{\mathfrak{a}}\left|c^{-2}(a d-b c) y\right|_{p}^{-1}-\mu_{\mathfrak{a}}\right) e_{\infty}\left(\left|c^{-2}(a d-b c) y\right|_{p}^{-1} j\right) \widetilde{\chi}_{\text {idelic }}\left(k_{0}\right), \\
\text { if } m_{\mathfrak{a}}\left|c^{-2}(a d-b c) y\right|_{p}^{-1}-\mu_{\mathfrak{a}} \in \mathbb{Z},
\end{array}\right. \\
& \text { otherwise. }
\end{aligned}
$$

If $c^{-1} d \notin \mathbb{Z}_{p}$

$$
\left(\begin{array}{cc}
0 & -1 \\
1 & 0
\end{array}\right)\left(\begin{array}{cc}
1 & c^{-1} d \\
0 & 1
\end{array}\right)=\left(\begin{array}{cc}
c^{-1} d & 0 \\
0 & c^{-1} d
\end{array}\right)\left(\begin{array}{cc}
c^{2} d^{-2} & -c d^{-1} \\
0 & 1
\end{array}\right)\left(\begin{array}{cc}
1 & 0 \\
c d^{-1} & 1
\end{array}\right)
$$

and $\left(\begin{array}{cc}1 & 0 \\ c d^{-1} & 1\end{array}\right) \in G L\left(2, \mathbb{Z}_{p}\right)$ since $c d^{-1} \in p \mathbb{Z}_{p}$. It follows that

$$
\begin{aligned}
& \left(\pi_{p}^{\prime}\left(\left(\begin{array}{cc}
0 & -1 \\
1 & 0
\end{array}\right)\left(\begin{array}{cc}
1 & c^{-1} d \\
0 & 1
\end{array}\right)\right) \cdot \varphi_{p}\right)\left(c^{-2}(a d-b c) y\right) \\
& \quad=\chi_{p}\left(c^{-1} d\right) e_{p}\left(-c^{-1} d^{-1}(a d-b c) y\right)\left(\pi_{p}^{\prime}\left(\left(\begin{array}{cc}
1 & 0 \\
c d^{-1} & 1
\end{array}\right)\right) \cdot \varphi_{p}\right)\left(d^{-2}(a d-b c) y\right)
\end{aligned}
$$

and

$$
\left(\pi_{p}^{\prime}\left(\left(\begin{array}{cc}
1 & 0 \\
c d^{-1} & 1
\end{array}\right)\right) \cdot \varphi_{p}\right)\left(d^{-2}(a d-b c) y\right)=W_{f, p}\left(\left(\begin{array}{cc}
d^{-2}(a d-b c) y & 0 \\
0 & 1
\end{array}\right)\left(\begin{array}{cc}
1 & 0 \\
c d^{-1} & 1
\end{array}\right)\right) .
$$


There exists a cusp $\mathfrak{a} \in \mathbb{Q} \cup\{\infty\}$, an integer $0 \leq j<m_{\mathfrak{a}}$ and $k_{0} \in K_{0}(N)$, which are uniquely determined by $g=\left(\begin{array}{ll}a & b \\ c & d\end{array}\right)$ and $y$ such that

$$
i_{\text {finite }}\left(\gamma_{\mathfrak{a}}\left(\begin{array}{ll}
1 & j \\
0 & 1
\end{array}\right)\right) i_{p}\left(\left(\begin{array}{cc}
\left|d^{-2}(a d-b c) y\right|_{p} d^{-2}(a d-b c) y & 0 \\
0 & 1
\end{array}\right)\left(\begin{array}{cc}
1 & 0 \\
c d^{-1} & 1
\end{array}\right)\right)=k_{0} \in K_{0}(N) .
$$

It follows that

$$
\begin{aligned}
& W_{f, p}\left(\left(\begin{array}{cc}
d^{-2}(a d-b c) y & 0 \\
0 & 1
\end{array}\right)\left(\begin{array}{cc}
1 & 0 \\
c d^{-1} & 1
\end{array}\right)\right) \\
& =\left\{\begin{array}{cc}
A\left(\mathfrak{a}, m_{\mathfrak{a}}\left|d^{-2}(a d-b c) y\right|_{p}^{-1}-\mu_{\mathfrak{a}}\right) e_{\infty}\left(\left|d^{-2}(a d-b c) y\right|_{p}^{-1} j\right) \widetilde{\chi}_{\text {idelic }}\left(k_{0}\right), \\
\text { if } m_{\mathfrak{a}}\left|d^{-2}(a d-b c) y\right|_{p}^{-1}-\mu_{\mathfrak{a}} \in \mathbb{Z}, \\
0, & \text { otherwise. }
\end{array}\right.
\end{aligned}
$$

Therefore, if $c^{-1} d \notin \mathbb{Z}_{p}$ then

$$
\begin{aligned}
& \pi_{p}^{\prime}\left(\left(\begin{array}{ll}
a & b \\
c & d
\end{array}\right)\right) \cdot \varphi_{p}(y) \\
& \quad=\left\{\begin{array}{c}
\chi_{p}(c) e_{p}\left(a c^{-1} y\right) A\left(\mathfrak{a}, m_{\mathfrak{a}}\left|d^{-2}(a d-b c) y\right|_{p}^{-1}-\mu_{\mathfrak{a}}\right) e_{\infty}\left(\left|d^{-2}(a d-b c) y\right|_{p}^{-1} j\right) \widetilde{\chi}_{\text {idelic }}\left(k_{0}\right), \\
\text { if } m_{\mathfrak{a}}\left|d^{-2}(a d-b c) y\right|_{p}^{-1}-\mu_{\mathfrak{a}} \in \mathbb{Z}, \\
0, \quad \text { otherwise. }
\end{array}\right.
\end{aligned}
$$

For fixed $\left(\begin{array}{ll}a & b \\ c & d\end{array}\right) \in G L\left(2, \mathbb{Q}_{p}\right)$ with $c \neq 0$, the function $\pi_{p}^{\prime}\left(\left(\begin{array}{ll}a & b \\ c & d\end{array}\right)\right) \cdot \varphi_{p} \in S\left(\mathbb{Q}_{p}^{\times}\right)$if and only if there exists an integer $M \geq 0$ such that $\pi_{p}^{\prime}\left(\left(\begin{array}{ll}a & b \\ c & d\end{array}\right)\right) \cdot \varphi_{p}(y)=0$ for $y \in p^{M} \mathbb{Z}_{p}$. If $\mu_{\mathfrak{a}} \neq 0$ then $A\left(\mathfrak{a}, m_{\mathfrak{a}}|y|_{p}^{-1}-\mu_{\mathfrak{a}}\right) \in S\left(\mathbb{Q}_{p}^{\times}\right)$ already. Therefore, for each cusp $\mathfrak{a} \in \mathbb{Q} \cup\{\infty\}$ with $\mu_{\mathfrak{a}}=0$, if there exists a non-negative integer $M_{\mathfrak{a}}$ such that $A\left(\mathfrak{a}, m_{\mathfrak{a}} p^{m}\right)=0$ for any integer $m \geq M_{\mathfrak{a}}$, then by the above computations, $\pi_{p}^{\prime}\left(\left(\begin{array}{ll}a & b \\ c & d\end{array}\right)\right) \cdot \varphi_{p} \in S\left(\mathbb{Q}_{p}^{\times}\right)$for any $\left(\begin{array}{ll}a & b \\ c & d\end{array}\right) \in G L\left(2, \mathbb{Q}_{p}\right)$.

Now assume that there exists a cusp $\mathfrak{a} \in \mathbb{Q} \cup\{\infty\}$ with $\mu_{\mathfrak{a}}=0$ such that for any non-negative integer $M$, there exists an integer $m \geq M$ and $A\left(\mathfrak{a}, m_{\mathfrak{a}} p^{m}\right) \neq 0$. By Theorem 15,

$$
A\left(\mathfrak{a}, m_{\mathfrak{a}} p^{m}\right)=\prod_{q \mid N} W_{f, q}\left(\left(\begin{array}{cc}
p^{m} & 0 \\
0 & 1
\end{array}\right) \gamma_{\mathfrak{a}}^{-1}\right) \neq 0 .
$$

So for any non-negative integer $M$ there exists an integer $m \geq M$ such that $W_{f, p}\left(\left(\begin{array}{cc}p^{m} & 0 \\ 0 & 1\end{array}\right) \gamma_{\mathfrak{a}}^{-1}\right)=\left(\pi_{p}^{\prime}\left(\gamma_{\mathfrak{a}}^{-1}\right) \cdot \varphi_{p}\right)\left(p^{m}\right) \neq$ 0 . Therefore

Then $\left(\pi_{p}, V_{p}\right)$ is not supercuspidal.

$$
\pi_{p}^{\prime}\left(\gamma_{\mathfrak{a}}^{-1}\right) \cdot \varphi_{p}(y) \notin S\left(\mathbb{Q}_{p}^{\times}\right) .
$$

Corollary 45. Let $f$ be a normalized Maass-Hecke newform of weight $k$, type $\nu$, level $N$, and character $\chi$ $(\bmod N)$ where $\chi$ is a primitive Dirichlet character. Let $f_{\text {adelic }}$ be the adelic lift of $f$ as in (8).

For a prime $p$, let $\left(\pi_{p}, V_{p}\right)$ be an irreducible and admissible representation of $G L\left(2, \mathbb{Q}_{p}\right)$. If $\left(\pi_{p}, V_{p}\right)$ is isomorphic to the local component of the irreducible global automorphic representation of $G L(2, \mathbb{A})$ (which is generated by $\left.f_{\text {adelic }}\right)$, then $\left(\pi_{p}, V_{p}\right)$ cannot be supercuspidal.

Proof. If $p \mid N$ and $\chi$ is primitive, we know that $A(\infty, p) \neq 0$ by [14. Since $f$ is a Maass-Hecke newform, for any positive integer $m, A\left(\infty, p^{m}\right)=A(\infty, p)^{m} \neq 0$. By Theorem [4], $\left(\pi_{p}, V_{p}\right)$ cannot be supercuspidal.

Remark: In the proof of Theorem 44 we say that if $\left(\pi_{p}, V_{p}\right)$ is supercuspidal then $p \mid N$. In fact, more it true: it follows directly from [5] that if $\left(\pi_{p}, V_{p}\right)$ is supercuspidal then $p^{2} \mid N$. For the convenience of the reader we briefly show how to deduce this fact from [5]. We shall assume the reader is familiar with the notation of [5] 
for the remainder of this paragraph. Suppose that the field $k$ considered in $[5]$ is $\mathbb{Q}_{p}$ and the representation $\varrho$ considered in Theorem 1 of [5] comes from a Maass form of level $N$, such that $p^{\alpha} \mid N$ and $p^{\alpha+1} \nmid N$. Then Casselman's conductor $c(\varrho)$ is the ideal $p^{\alpha} \cdot \mathbb{Z}_{p}$. It is also shown in [5] (page 304, line 16) that in the supercuspidal case $c(\varrho)=p^{-n_{1}}$ where $n_{1}$ is a certain integral invariant of the representation $\varrho$ which was shown by Jacquet-Langlands to be at most -2 . (See [5], p. 303, paragraphs 1 and 2.) Thus $\alpha=-n_{1} \geq 2$.

\section{Toward a Classical Proof of Theorem [15]}

In this section we study the problem of giving a proof of Theorem [15] in purely classical terms, with a partial result in this direction being given in Theorem 49 below. It is helpful to first review the classical proof of multiplicativity of Fourier coefficients at infinity. Suppose that

$$
\lambda \cdot f(z)=\left(T_{p}^{\chi} f\right)(z):=\frac{1}{\sqrt{p}}\left(\chi(p) f(p z)+\sum_{b=0}^{p-1} f\left(\frac{z+i}{p}\right)\right),
$$

for some $\lambda \in \mathbb{C}$ and all $z \in \mathfrak{h}$. Suppose further that

$$
f(z)=\sum_{n \neq 0} A(\infty, n) W_{\frac{\operatorname{sgn}(n) k}{2}, \nu-\frac{1}{2}}(4 \pi|n| \cdot y) e^{2 \pi i n x} .
$$

Now plug (47) into (46), using the identity

$$
\sum_{b=0}^{p-1} e^{\frac{2 \pi i n b}{p}}= \begin{cases}p & \text { if } p \mid n \\ 0 & \text { if } p \nmid n\end{cases}
$$

By comparing coefficients of $\frac{W_{\operatorname{sgn}(n) k}, \nu-\frac{1}{2}}{2}(4 \pi|n| \cdot y) e^{2 \pi i n x}$ in (46), we see that

$$
\frac{\chi(p)}{\sqrt{p}} A\left(\infty, \frac{n}{p}\right)-\lambda A(\infty, n)+\sqrt{p} A(\infty, n p)=0,
$$

with the understanding that $A\left(\infty, \frac{n}{p}\right)=0$ if $p \nmid n$. It follows that for all $n$ with $\operatorname{gcd}(n, p)=1$, and all $k \geq 0$, the coefficient $A\left(\infty, n p^{k}\right)=b_{k} \cdot A(\infty, n)$, where $\left(b_{k}\right)_{k=-1}^{\infty}$ is the unique sequence satisfying the recurrence relation

$$
\sqrt{p} b_{k}-\lambda b_{k-1}+\frac{\chi(p)}{\sqrt{p}} b_{k-2}=0
$$

and the initial conditions $b_{-1}=0, b_{0}=1$.

It is natural to ask whether one may prove that the Fourier coefficients at cusps other than $\infty$ satisfy a recurrence relation analogous to (48). The answer, in general, appears to be "no." In fact, what we shall prove in Theorem 49 below is a formula which, in general, involves Fourier coefficients at three different cusps.

Theorem 49. Let $f$ be a Maass form of weight $k$ level $N$ and character $\chi$. Assume that a maximal set of $\Gamma_{0}(N)$-inequivalent cusps and a matrix $\gamma_{\mathfrak{a}}$ for each cusp $\mathfrak{a}$ in this set have been chosen as in section 5 , Let $A(\mathfrak{a}, n)$ denote the Fourier coefficients defined using the matrices $\sigma_{\mathfrak{a}}$ determined by these choices of $\mathfrak{a}, \gamma_{\mathfrak{a}}$. Let $p$ be a prime which does not divide $N$, and assume that $T_{p}^{\chi} f=\lambda f$. Then for each representative cusp $\mathfrak{a}$ there exist cusps $\mathfrak{a}^{\prime}$ and $\mathfrak{a}^{\prime \prime}$, and integers $j^{\prime}, j^{\prime \prime}, \lambda^{\prime}, \lambda^{\prime \prime}$, such that

$$
\begin{aligned}
\frac{\chi\left(\lambda^{\prime \prime}\right)}{\sqrt{p}} A\left(\mathfrak{a}^{\prime \prime}, \frac{n+\mu_{\mathfrak{a}}}{p}-\right. & \left.\mu_{\mathfrak{a}^{\prime \prime}}\right) e\left(j^{\prime \prime}\left(n+\mu_{\mathfrak{a}}\right)\right)-\lambda A(\mathfrak{a}, n) \\
& +\sqrt{p} \chi\left(\lambda^{\prime}\right) e\left(j^{\prime} \frac{n+\mu_{\mathfrak{a}}}{p}\right) A\left(\mathfrak{a}^{\prime},\left\lfloor p \cdot\left(n+\mu_{\mathfrak{a}}\right)\right\rfloor\right)=0,
\end{aligned}
$$

with the caveat that $\frac{n+\mu_{\mathfrak{a}}}{p}-\mu_{\mathfrak{a}^{\prime \prime}}$ need not be integral, and the convention that if it is not then $A\left(\mathfrak{a}^{\prime \prime}, \frac{n+\mu_{\mathfrak{a}}}{p}-\mu_{\mathfrak{a}^{\prime \prime}}\right)$ is defined to be zero. The cusps $\mathfrak{a}^{\prime}$, and $\mathfrak{a}^{\prime \prime}$ are $p \cdot \mathfrak{a}$ and $p^{-1} \cdot \mathfrak{a}$, respectively, in terms of the action of $\mathbb{Z} / N \mathbb{Z}$ on a set of cusp representatives satisfying our hypotheses which was defined at the end of the last section. 
For prime power $q^{e}$, the values of $\lambda^{\prime}, \lambda^{\prime \prime}$, are given in the table below. The integers $j^{\prime}, j^{\prime \prime}$, are both zero unless $\mathfrak{a}=\frac{1}{c_{1} q^{l}}$ with $l<\frac{e}{2}$. In that case, we have

$$
\begin{aligned}
& 0 \leq j^{\prime}, j^{\prime \prime}<q^{e-l}, \quad 0 \leq c_{1}^{\prime}, c_{1}^{\prime \prime}<q^{l}, \\
& c_{1}^{\prime \prime} p \equiv c_{1} \quad\left(\bmod q^{l}\right), \quad c_{1}^{\prime} \equiv c_{1} p \quad\left(\bmod q^{l}\right), \\
& c_{1}^{\prime \prime}\left(p-j^{\prime \prime} q^{l} c_{1}\right) \equiv c_{1} \quad(\bmod q)^{e}, \quad\left(1-q^{l} c_{1} j^{\prime} p\right) c_{1}^{\prime} \equiv c_{1} p \quad\left(\bmod q^{e}\right) .
\end{aligned}
$$

For $N=\prod_{q \in S} q^{e_{q}}$, the integer $\lambda^{\prime}$ may be taken to be the smallest positive solution to the system of congruences: $\lambda^{\prime} \equiv \lambda_{q}^{\prime}\left(\bmod q^{e_{q}}\right),(\forall q \in S)$, where, for each $q$, the integer $\lambda_{q}^{\prime}$ is obtained by applying the result to the prime power $q^{e_{q}}$ and the cusp $\mathfrak{a}_{q}$ for $\Gamma_{0}\left(q^{e_{q}}\right)$ such that $\gamma_{\mathfrak{a}} \equiv \gamma_{\mathfrak{a}_{q}}\left(\bmod q^{e_{q}}\right)$. The values of $j^{\prime}, j^{\prime \prime}, \lambda^{\prime \prime}$ are obtained similarly.

(Inverses taken modulo $q^{e}$.)

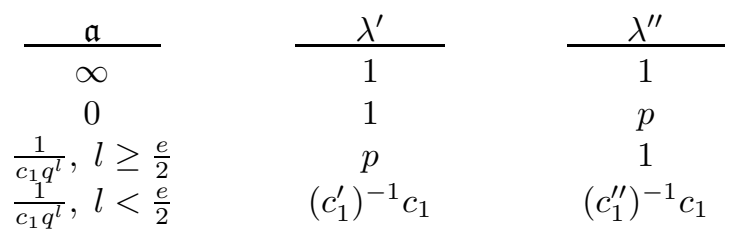

Remarks: If $\chi$ is not primitive, it may not be necessary for $\lambda^{\prime}$ to be congruent to $\lambda_{q}^{\prime}$ modulo $q^{e_{q}}$; a lower power of $q$ may suffice. The same applies to $\lambda^{\prime \prime}$. It will turn out that the systems of congruences for $j^{\prime}$ and $j^{\prime \prime}$ can always be taken modulo properly lower powers of $q$. (See $e_{q}^{\prime}$ and $N^{\prime}$ in the statement of Proposition 50.)

Theorem 49 follows easily from the following proposition, by the same argument sketched in the classical case at the beginning of this section. This proposition works with the group algebra $\mathbb{C}\left[G L(2, \mathbb{Q})^{+}\right]$. The action of $G L(2, \mathbb{Q})^{+}$on functions $\mathfrak{h} \rightarrow \mathbb{C}$ by the weight $k$ slash operator $\left.\right|_{k}$ extends by $\mathbb{C}$-linearity to an action of $\mathbb{C}\left[G L(2, \mathbb{Q})^{+}\right]$, and this identifies the Hecke operator $T_{p}^{\chi}$ with

$$
\chi(p)\left(\begin{array}{ll}
p & 0 \\
0 & 1
\end{array}\right)+\sum_{b=0}^{p-1}\left(\begin{array}{ll}
1 & b \\
0 & p
\end{array}\right) \in \mathbb{C}\left[G L(2, \mathbb{Q})^{+}\right] .
$$

We shall also consider the right ideal in $\mathbb{C}\left[G L(2, \mathbb{Q})^{+}\right]$generated by all $\gamma-\chi(\gamma)$ with $\Gamma \in \Gamma_{0}(N)$. It is easy to see that any modular form of character $\chi$ for $\Gamma_{0}(N)$ is annihilated by this ideal.

Proposition 50. Fix a cusp $\mathfrak{a}$ and let $\mathfrak{a}^{\prime}, \mathfrak{a}^{\prime \prime}, \lambda^{\prime}, \lambda^{\prime \prime}, j^{\prime}, j^{\prime \prime}$ be defined as in Theorem 49 . For each $q \in S$, let

$$
e_{q}^{\prime}= \begin{cases}0, & \mathfrak{a}_{q}=\infty \\ e_{q}, & \mathfrak{a}_{q}=0 \\ e_{q}-l, & \mathfrak{a}_{q}=\frac{1}{c q^{\nu}}\end{cases}
$$

Let $N^{\prime}=\prod_{q \in S} q^{e_{q}^{\prime}}$. Let $\mathcal{I}$ denote the right ideal in the group ring $\mathbb{C}\left[G L(2, \mathbb{Q})^{+}\right]$generated by all $\gamma-\chi(\gamma), \gamma \in$ $\Gamma_{0}(N)$. Then

$$
T_{p}^{\chi} \gamma_{\mathfrak{a}} \equiv \chi\left(\lambda^{\prime \prime}\right) \gamma_{\mathfrak{a}^{\prime \prime}}\left(\begin{array}{ll}
p & 0 \\
0 & 1
\end{array}\right)\left(\begin{array}{cc}
1 & j^{\prime \prime} \\
0 & 1
\end{array}\right)+\chi\left(\lambda^{\prime}\right) \gamma_{\mathfrak{a}^{\prime}} \sum_{\substack{0 \leq b<N^{\prime} p \\
b \equiv j^{\prime}}}\left(\begin{array}{ll}
1 & b \\
0 & p
\end{array}\right) \quad\left(\bmod N^{\prime}\right)
$$

As a first step towards the proof of Proposition 50, we show the following.

Lemma 51. For each element of the set $\left\{(c, d) \in(\mathbb{Z} / N \mathbb{Z})^{2} \mid\langle c, d\rangle=\mathbb{Z} / N \mathbb{Z}\right\}$, fix an element $s(c, d)$ of $S L(2, \mathbb{Z})$ such that the bottom row of $s(c, d)$ is congruent to $(c, d)(\bmod N)$. Take $p$ a prime not dividing $N$. Then for any matrix $\gamma \in S L(2, \mathbb{Z})$ such that the bottom row of $\gamma$ is congruent to $(c, d)(\bmod N)$, we have

$$
T_{p}^{\chi} \gamma \equiv s(c, p d)\left(\begin{array}{ll}
p & 0 \\
0 & 1
\end{array}\right)+\sum_{i=0}^{p-1} s(p c, d-i c)\left(\begin{array}{ll}
1 & i \\
0 & p
\end{array}\right) \quad(\bmod \mathcal{I})
$$


where $\mathcal{I}$ is the right ideal of $\mathbb{C}\left[G L(2, \mathbb{Q})^{+}\right]$defined in Proposition 50.

Proof. Let

$$
S_{p}=\left\{\left(\begin{array}{ll}
p & 0 \\
0 & 1
\end{array}\right)\right\} \cup\left\{\left(\begin{array}{ll}
1 & i \\
0 & p
\end{array}\right): 0 \leq i \leq p\right\}
$$

Then, for all $\xi \in S_{p}$,

$$
S L(2, \mathbb{Z}) \cdot \xi \cdot S L(2, \mathbb{Z})=\coprod_{\xi^{\prime} \in S_{p}} S L(2, \mathbb{Z}) \cdot \xi^{\prime}=\coprod_{\xi^{\prime} \in S_{p}} \xi^{\prime} \cdot S L(2, \mathbb{Z}) .
$$

It follows that for all $\xi \in S_{p}, \gamma \in S L(2, \mathbb{Z})$ there exist unique $\xi^{\prime} \in S_{p}, \gamma^{\prime} \in S L(2, \mathbb{Z})$ such that $\xi \gamma=\gamma^{\prime} \xi^{\prime}$, and that, for fixed $\gamma$, the map $\xi \mapsto \xi^{\prime}$ is a bijection $S_{p} \rightarrow S_{p}$. If $\xi$ and $\gamma$ are given, then the element $\xi^{\prime}$ may be described concretely as the unique element of $S_{p}$ such that $\xi \gamma\left(\xi^{\prime}\right)^{-1} \in S L(2, \mathbb{Z})$.

Fix any $\gamma_{0} \in \Gamma_{0}(N)$ such that $\widetilde{\chi}\left(\gamma_{0}\right)=\chi(p)$, and let

$$
S_{p}^{\prime}=\left\{\gamma_{0}\left(\begin{array}{ll}
p & 0 \\
0 & 1
\end{array}\right)\right\} \cup\left\{\left(\begin{array}{ll}
1 & i \\
0 & p
\end{array}\right): 0 \leq i \leq p\right\} .
$$

Then

$$
T_{p}^{\chi}=\sum_{\xi \in S_{p}^{\prime}} \xi(\bmod \mathcal{I}) .
$$

To prove that two individual matrices in $G L(2, \mathbb{Q})^{+}$are equivalent $(\bmod \mathcal{I})$ it suffices to prove that their bottom rows are congruent modulo $N$. It is clear that the bottom row of $\xi s(c, d)$ is $(p c, p d)$ for all $\xi \in S_{p}^{\prime}$, so that the bottom row of $\xi s(c, d)\left(\xi^{\prime}\right)^{-1}$ is the row vector $(p c, p d) \cdot\left(\xi^{\prime}\right)^{-1}$. As $\xi$ ranges over $S_{p}^{\prime}$, this row vector ranges over the set $\{(p c, d-i c)\} \cup\{(c, p d)\}$. This completes the proof.

Proof of Proposition 50; We assume that we have fixed a maximal set of $\Gamma_{0}(N)$-inequivalent cusps together with a choice of matrix $\gamma_{\mathfrak{b}}$ for each cusp $\mathfrak{b}$ in this set as in section 5 , Let

$$
X=\left\{(c, d) \in(\mathbb{Z} / N \mathbb{Z})^{2} \mid\langle c, d\rangle=\mathbb{Z} / N \mathbb{Z}\right\} .
$$

For any $x=\left(x_{0}, x_{1}\right) \in X$, there exist a unique cusp $\mathfrak{b}$ from our fixed set of representatives, corresponding to the $\Gamma_{\infty}$-orbit of $\left[x_{0}: x_{1}\right]$ in $\mathbb{P}^{1}(\mathbb{Z} / N \mathbb{Z})$. If $\gamma \in S L(2, \mathbb{Z})$ has bottom row $\equiv\left(x_{0}, x_{1}\right)(\bmod N)$, then it is possible to choose $\gamma_{0} \in \Gamma_{0}(N)$ and $j \in \mathbb{Z}$ such that $\gamma=\gamma_{0} \gamma_{\mathfrak{b}}\left(\begin{array}{ll}1 & j \\ 0 & 1\end{array}\right)$. It follows immediately that $\gamma \equiv \tilde{\chi}\left(\gamma_{0}\right) \gamma_{\mathfrak{b}}\left(\begin{array}{ll}1 & j \\ 0 & 1\end{array}\right)$ $(\bmod \mathcal{I})$. It is possible to make $\gamma_{0}$ and $j$ unique by requiring $0 \leq j \leq m_{\mathfrak{b}}$, but we will not do so. It turns out that it is sometimes possible to gain a bit of control over $\widetilde{\chi}\left(\gamma_{0}\right)$ by allowing $j$ to vary over a larger range, and this approach is more convenient.

In order to prove Proposition 50, we must carry out this analysis carefully for each element of the set $\{(p c, d-i c)\} \cup\{(c, p d)\}$, as $(c, d)$ ranges over the bottom rows of the matrices $\gamma_{\mathfrak{a}}$. We first complete the case when $N=q^{e}$. In this case, the pairs $(c, d)$ considered are $(1,0),(0,1)$, and $\left(c q^{l}, 1\right)$ for $0<l<e, 0<c<q^{e-l}$ and $q \nmid c$. Let us first look carefully at $(p c, d-i c)$ in the case when $(c, d)=(1,0)$. clearly $(p c, d-i c)=(p,-i)$ in this case. The corresponding element of $\mathbb{P}^{1}\left(\mathbb{Z} / q^{e} \mathbb{Z}\right)$ is $[p:-i]$, or $[1:-i \bar{p}]$, where $\bar{p}$ denotes the inverse modulo $q^{e}$. This element of $\mathbb{P}^{1}\left(\mathbb{Z} / q^{e} \mathbb{Z}\right)$ lies in the $\Gamma_{\infty}$-orbit for which the standard representative is $[1: 0]$. This tells us that $\gamma_{\mathfrak{b}}$ in this case is $\left(\begin{array}{cc}0 & -1 \\ 1 & 0\end{array}\right)$. Furthermore, since $[1:-i \bar{p}]=\left[\begin{array}{cc}1: 0 & 0\end{array} \cdot\left(\begin{array}{cc}1 & -i \cdot \bar{p} \\ 0 & 1\end{array}\right)\right.$, we see that $j$ may be taken to be $-i \cdot \bar{p}$. (Here, we interpret this as taking the additive inverse and product modulo $q^{e}$, so that the answer is an integer between 0 and $q^{e}-1$, inclusive.)

In order to keep track of $\widetilde{\chi}\left(\gamma_{0}\right)$ as well, it is necessary to work with $X$ rather than $\mathbb{P}^{1}$. The more precise statement is that $(p,-i)=p \cdot(1,0) \cdot\left(\begin{array}{cc}1 & -i \bar{p} \\ 0 & 1\end{array}\right)$. This immediately implies that

$$
\left(\begin{array}{cc}
* & * \\
p & -i
\end{array}\right)=\gamma_{0} \cdot\left(\begin{array}{cc}
0 & -1 \\
1 & 0
\end{array}\right) \cdot\left(\begin{array}{cc}
1 & -i \bar{p} \\
0 & 1
\end{array}\right),
$$

with $\gamma_{0} \in \Gamma_{0}\left(q^{e}\right)$ such that $\tilde{\chi}\left(\gamma_{0}\right)=\chi(p)$. Hence

$$
\left(\begin{array}{cc}
* & * \\
p & -i
\end{array}\right) \equiv \chi(p) \cdot\left(\begin{array}{cc}
0 & -1 \\
1 & 0 \\
27
\end{array}\right) \cdot\left(\begin{array}{cc}
1 & -i \bar{p} \\
0 & 1
\end{array}\right) \quad(\bmod \mathcal{I})
$$


in this case. Now, $\left(\begin{array}{cc}1 & -i \bar{p} \\ 0 & 1\end{array}\right) \cdot\left(\begin{array}{ll}1 & i \\ 0 & p\end{array}\right)=\left(\begin{array}{cc}1 & i-i p \bar{p} \\ 0 & p\end{array}\right)$. Clearly, for each $i$, the integer $i-i p \bar{p}$ is divisible by $q^{e}$. Furthermore, these integers are all distinct modulo $p$, and all in the range from 0 to $p q^{e}-1$. It follows that we have

$$
\sum_{i=0}^{p-1}\left(\begin{array}{cc}
* & * \\
p & -i
\end{array}\right) \cdot\left(\begin{array}{cc}
1 & i \\
0 & p
\end{array}\right) \equiv \chi(p) \cdot\left(\begin{array}{cc}
0 & -1 \\
1 & 0
\end{array}\right) \cdot \sum_{\substack{0 \leq b \leq q^{e} p \\
b \equiv 0}}\left(\begin{array}{ll}
1 & b \\
0 & p
\end{array}\right) .
$$

Having worked this case in detail to illustrate the method, we shall henceforth be more brief. To finish the case $(1,0)$ we simply note that if $(c, d)=(1,0)$ then $(c, p d)$ is again $(1,0)$, whence a contribution of $\left(\begin{array}{cc}* & * \\ 1 & 0\end{array}\right) \equiv\left(\begin{array}{cc}0 & -1 \\ 1 & 0\end{array}\right)(\bmod \mathcal{I})$. This completes the proof of Proposition 50 in the case $N=q^{e}$ and $\mathfrak{a}=0$. The case $\mathfrak{a}=\infty$ is, of course, trivial.

We turn to $\mathfrak{a}=\frac{1}{c_{1} q^{l}}$. First, consider. $\left(c_{1} q^{l}, p\right)$. We must identify the standard representative for the orbit of this element under the action of $\mathbb{Z} / N \mathbb{Z} \times \Gamma_{\infty}$, where $\mathbb{Z} / N \mathbb{Z}$ acts by scalar multiplication and $\Gamma_{\infty}$ acts by matrix multiplication on the right. Referring to Lemma 16, it is not difficult to see that the standard representative is $\left(c_{1}^{\prime \prime} q^{l}, 1\right)$ where $c_{1}^{\prime \prime}$ is uniquely determined by the conditions that $p c_{1}^{\prime \prime} \equiv c_{1}\left(\bmod q^{e-l}\right)$ and $0<c_{1}^{\prime \prime}<q^{\min (l, e-l)}$. In the simpler case $l \geq e-l$, we get

$$
\left(\begin{array}{cc}
* & * \\
c_{1} q^{l} & p
\end{array}\right) \equiv \chi(p)\left(\begin{array}{cc}
1 & 0 \\
c_{1}^{\prime \prime} q^{l} & 1
\end{array}\right) \quad(\bmod \mathcal{I})
$$

If $l<e-l$, it is a bit more complicated. Because we deal with reduction and inversion modulo various powers of $q$, it is convenient to introduce a more explicit notation. For $a, r$ integers with $r$ positive, let $[a]_{r}$ denote the unique integer between 0 and $q^{r}-1$, inclusive, which is congruent to $a\left(\bmod q^{r}\right)$ and $[a]_{r}^{-1}$ the unique integer in the same range such that $a \cdot[a]_{r}^{-1} \equiv 1\left(\bmod q^{r}\right)$.

When $l<e-l$, we have $c_{1}^{\prime \prime}=c_{1}\left[[p]_{e-l}^{-1}\right]_{l}$. It is necessary to choose $j^{\prime \prime}$ such that $c_{1}\left[p-j^{\prime \prime} c_{1} q^{l}\right]_{e-l}^{-1} \equiv c_{1}^{\prime \prime}$ $\left(\bmod q^{e-l}\right)$. However, it is possible, and more convenient, to choose $j^{\prime \prime}$ subject to the more stringent condition that $c_{1}\left[p-j^{\prime \prime} c_{1} q^{l}\right]_{e}^{-1} \equiv c_{1}^{\prime \prime}\left(\bmod q^{e}\right)$. This ensures that $\chi\left(p-j^{\prime \prime} c_{1} q^{l}\right)=\chi\left(c_{1}\right) \chi\left(c_{1}^{\prime \prime}\right)^{-1}$. We have

$$
\left(c_{1} q^{l}, p\right)=\left(c_{1} q^{l}, p-j^{\prime \prime} c_{1} q^{l}\right)\left(\begin{array}{cc}
1 & j^{\prime \prime} \\
0 & 1
\end{array}\right)=\left(p-j^{\prime \prime} c_{1} q^{l}\right)\left(c_{1}^{\prime \prime} q^{l}, 1\right)\left(\begin{array}{cc}
1 & j^{\prime \prime} \\
0 & 1
\end{array}\right),
$$

and deduce

$$
\left(\begin{array}{cc}
* & * \\
c_{1} q^{l} & p
\end{array}\right) \equiv \chi\left(c_{1}\right) \chi\left(c_{1}^{\prime \prime}\right)^{-1}\left(\begin{array}{cc}
1 & 0 \\
c_{1}^{\prime \prime} q^{l} & 1
\end{array}\right)\left(\begin{array}{cc}
1 & j^{\prime \prime} \\
0 & 1
\end{array}\right) .
$$

Now, consider $\left(c_{1} p q^{l}, 1-i c_{1} q^{l}\right)$. When $l \geq e-l$ the most expedient thing is to write this as

$$
\left(c_{1} p q^{l}, 1\right)\left(\begin{array}{cc}
1 & {\left[-[p]_{e-l}^{-1} \cdot i\right]_{e-l}} \\
0 & 1
\end{array}\right) .
$$

Much as above, we get

$$
\sum_{i=0}^{p-1}\left(\begin{array}{cc}
* & * \\
c_{1} p q^{l} & 1-i c_{1} q^{l}
\end{array}\right) \equiv\left(\begin{array}{cc}
1 & 0 \\
c_{1}^{\prime} q^{l} & 1
\end{array}\right) \sum_{\substack{0 \leq b<p q^{e-l} \\
b \equiv 0}}\left(\begin{array}{ll}
1 & b \\
0 & p
\end{array}\right) .
$$

When $l<e-l$ we choose $j(i)$ so that $c_{1} p\left[1-i c_{1} q^{l}-j(i) c_{1} p q^{l}\right]_{e}^{-1} \equiv c_{1}^{\prime}\left(\bmod q^{e}\right)$. It is easy to see that this has a unique solution modulo $q^{e-l}$, and that the quantity $i+j(i) p$ is constant, modulo $q^{e-l}$, as $i$ varies. We get

$$
\sum_{i=0}^{p-1}\left(\begin{array}{cc}
* & * \\
c_{1} p q^{l} & 1-i c_{1} q^{l}
\end{array}\right) \equiv \sum_{\substack{0 \leq b<p q^{e-l} \\
b \equiv j(0)}}\left(\begin{array}{ll}
1 & b \\
0 & p
\end{array}\right) .
$$

It is easily verified that $j(0)=j^{\prime}$, defined as in the statement of the theorem. This completes the proof in the prime power case.

To complete the general case, let us resume the notation $N=\prod_{q \in S} q^{e_{q}}$. Take $(c, d)$ the bottom row of the matrix $\gamma_{\mathfrak{a}}$ corresponding to some cusp $\mathfrak{a}$, and consider $(c, p d)$. For each $q$, we know that $(c, p d)$ is equivalent modulo $q^{e_{q}}$ to one of the row vectors for which the analysis was carried out above. Thus, for each $q$ we obtain 
a cusp $\mathfrak{a}_{q}^{\prime \prime}, j_{q}^{\prime \prime} \in \mathbb{Z}$ and $\lambda_{q}^{\prime \prime} \in\left(\mathbb{Z} / q^{e_{q}} \mathbb{Z}\right)^{\times} \operatorname{such}$ that $(c, p d) \equiv \lambda_{q}^{\prime \prime} \cdot\left(c_{q}^{\prime}, d_{q}^{\prime}\right) \cdot\left(\begin{array}{cc}1 & j_{q}^{\prime} \\ 0 & 1\end{array}\right)\left(\bmod q^{e_{q}}\right)$, where $\left(c_{q}^{\prime}, d_{q}^{\prime}\right)$ is the bottom row of $\gamma_{\mathfrak{a}_{q}^{\prime \prime}}$. Solving systems of congruences, we obtain a cusp $\mathfrak{a}^{\prime \prime}$, integer $j^{\prime \prime}$ and element $\lambda^{\prime \prime}$ of $(\mathbb{Z} / N \mathbb{Z})^{\times}$such that

$$
(c, p d)=\lambda^{\prime \prime}\left(c^{\prime}, d^{\prime}\right)\left(\begin{array}{cc}
1 & j^{\prime \prime} \\
0 & 1
\end{array}\right),
$$

where $\left(c^{\prime}, d^{\prime}\right)$ is the bottom row of $\gamma_{\mathfrak{a}^{\prime \prime}}$. It follows that

$$
\left(\begin{array}{cc}
* & * \\
c & p d
\end{array}\right) \equiv \chi\left(\lambda^{\prime \prime}\right) \gamma_{a^{\prime \prime}}\left(\begin{array}{cc}
1 & j^{\prime \prime} \\
0 & 1
\end{array}\right) \quad(\bmod \mathcal{I})
$$

Similarly, for $i=0$ to $p-1$, by passing to the individual primes, using the prime power case, and then solving systems of congruences, we obtain

$$
\left(\begin{array}{cc}
* & * \\
p c^{\prime} & d-i p c^{\prime}
\end{array}\right) \equiv \chi\left(\lambda^{\prime}\right) \gamma_{\mathfrak{a}^{\prime}}\left(\begin{array}{cc}
1 & j(i) \\
0 & 1
\end{array}\right) \quad(\bmod \mathcal{I}), \quad(0 \leq i<p)
$$

for some cusp $\mathfrak{a}^{\prime}$ some $\lambda^{\prime} \in(\mathbb{Z} / N \mathbb{Z})^{\times}$, which are independent of $i$, and some integers $j(i), 0 \leq i<p$. Furthermore, it follows from the analysis done in the prime power case above that $j(i) \cdot p+i \equiv j_{q}^{\prime}\left(\bmod q_{q}^{e^{\prime}}\right)$, for each $q$, where $e_{q}^{\prime}$ is defined as in the proposition and $j_{q}^{\prime}$ is as described (for prime powers) in the statement of Theorem 49, It follows at once that $j(i) \cdot p+i$ is constant $\left(\bmod N^{\prime}\right)$, where $N^{\prime}$ is defined as in the statement of Proposition 50, and that the value is obtained by solving the system of congruences obtained from the various $q$.

\section{REFERENCES}

[1] T. Asai, "On the Fourier coefficients of automorphic forms at various cusps and some applications to Rankin's convolution," J. Math. Soc. Japan 28 (1976), no. 1, 48-61.

[2] A.O.L. Atkin and J. Lehner, "Hecke operators on $\Gamma_{0}(m) . "$ Math. Ann. 185 (1970) 134-160.

[3] J. Bernstein, P. Deligne, D. Kazhdan, and M-F. Vignéras, "Representations des groupes sur un corps local." Hermann, Paris (1984).

[4] D. Bump, "Automorphic forms and representations." Cambridge University Press (1997).

[5] W. Casselman, "On some results of Atkin and Lehner." Math. Ann. 201 (1973), pp. 301-314.

[6] W. Casselman and J. Shalika, The unramified principal series of $p$-adic groups. II. The Whittaker function. Compositio Math. 41 (1980), no. 2, 207-231.

[7] D. Flath, "Decomposition of representations into tensor products." Borel and Casselman part 1 (1979), pp. 179-184.

[8] S. Gelbart, "Lectures on the Arthur-Selberg trace formula." University Lecture Series, 9, American Mathematical Society, Providence, RI (1996).

[9] S. Gelbart, "Three lectures on the modularity of $\bar{\rho}_{E, 3}$ and the Langlands reciprocity conjecture," in Modular forms and Fermat's last theorem (Boston, MA, 1995), 155-207, Springer, New York, 1997.

[10] R. Godement, Notes on Jacquet-Langlands theory, mimeographed notes, Institute for Advanced Study, Princeton, NJ (1970).

[11] D. Goldfeld, "Automorphic forms and $L$-functions for the Group $G L(n, \mathbb{R})$." Cambridge University Press (2006).

$[12]$ D. Goldfeld and J. Hundley, "Automorphic representations and L-functions for the group GL(n, $\mathbb{A})$." Cambridge University Press, to appear

[13] E. Kowalski, Y.-K. Lau, K. Soundararajan and J. Wu, "On modular signs," preprint, available at http://www.math.ethz.ch/ kowalski/papers-books.html\#signs

[14] H. Iwaniec, "Topics in classical automorphic forms." Graduate Studies in Mathematics Volume 17 (1997).

[15] H. Jacquet and R.P. Langlands, "Automorphic forms on GL(2)." Lecture Notes in Mathematics Vol. 114. Springer-Verlag, Berlin-New York (1970).

[16] A.A. Kirillov, "Infinite-dimensional unitary representations of a second-order matrix group with elements in a locally compact field." (Russian) Dokl. Akad. Nauk SSSR 150, 740-743 (1963).

[17] A.A. Kirillov, "Classification of irreducible unitary representations of a group of second-order matrices with elements from a locally compact field." (Russian) Dokl. Akad. Nauk SSSR 168 273-275; translated as Soviet Math. Dokl. 7, 628-631 (1966).

[18] H. Kojima, "Rankin's method in the case of level $4 q$ and its applications to the Doi-Naganuma lifting," Tôhoku Math. J. (2) 31, no. 2, 195-205 (1979).

[19] R. Langlands, Base change for GL(2), Annals of Mathematics Studies, 96. Princeton University Press, Princeton, N.J.; University of Tokyo Press, Tokyo, 1980

[20] W. Li, "Newforms and functional equations." Math. Ann. 212, 285-315 (1975).

[21] H. Maass, "Über automorphe Funktionen von mehreren Ver änderlichen und die Bestimmung von Dirichletschen Reihen durch Funktionalgleichungen." (German) Ber. Math.-Tagung Tübingen 1946 (1946), 100-102 (1947). 
[22] H. Maass, "Lectures on modular functions of one complex variable." Second edition, Tata Institute of Fundamental Research Lectures on Mathematics and Physics, 29, Tata Institute of Fundamental Research, Bombay, (1983).

[23] F. Shahidi, "A proof of Langlands' conjecture on Plancherel measures; complementary series for p-adic groups." Ann. of Math. (2) 132, no. 2, 273-330 (1990).

[24] F. Shahidi, "Symmetric power L-functions for GL(2)," in Elliptic curves and related topics, 159-182, CRM Proc. Lecture Notes, 4, Amer. Math. Soc., Providence, RI, 1994.

Mathematics Department, Columbia University, New York, NY 1002, and Department of Mathematics, Mailcode 4408, Southern Illinois University, 1245 Lincoln Drive, Carbondale, IL 62901 